\title{
Construction and justification of Paris-like fatigue laws from Dugdale-type cohesive models
}

\author{
Radhi Abdelmoula $\cdot$ Jean-Jacques Marigo • \\ Thibaut Weller
}

Received: 5 March 2010/Accepted: 13 July 2010/Published online: 28 August 2010

(C) Springer-Verlag 2010

\begin{abstract}
We propose a construction of fatigue laws from cohesive forces models in the case of a crack submitted to a mode I cyclic loading. Taking the cumulated opening as the memory variable and the surface energy density associated with Dugdale's model, we explicitly construct the fatigue law which gives the crack growth rate by cycle $d \ell / d N$ in terms of the stress intensity factor $K_{I}$. In particular, we recover a Paris law with an exponent 4, i.e., $d \ell l d N=C K_{I}^{4}$, when $K_{I}$ is small, the coefficient $C$ being explicitly expressed in terms of the material parameters. Furthermore, the law can be applied in the full range of values of $K_{I}$ and can be extended to non simple cycles.
\end{abstract}

Keywords Fatigue - Fracture mechanics .

Cohesive force - Cyclic loading · Variational approach . Complex analysis

\section{Introduction}

It is not possible to account for fatigue phenomenon, i.e., for propagation of a crack under cyclic loading, within

\footnotetext{
R. Abdelmoula

LPMTM (UPR-CNRS 9001), Université Paris XIII,

93140 Villetaneuse, France

e-mail: radhi@galilee.univ-paris13.fr

J.-J. Marigo ( $\square)$

Laboratoire de Mécanique des Solides (UMR 7649),

Ecole Polytechnique, 91128 Palaiseau, France

e-mail: marigo@lms.polytechnique.fr

T. Weller

LMGC (UMR 5508), Université Montpellier II,

Montpellier Cedex, France

e-mail: weller@1mgc.univ-montp2.fr
}

Griffith's theory (i.e., when the material behavior is purely elastic and the crack lips are free of cohesive forces), because the response is unchanged after the first cycle. That becomes possible with cohesive forces but only if a right irreversibility condition is introduced into the model. Indeed, it is essential that the cohesive forces depend on the sign of the rate of the displacement jump and not only on the current value of the displacement jump. Owing to this directional rate dependence, we obtain a response which differs in loading and unloading phases. Moreover, by introducing into the constitutive relation a memory variable which cumulates all the oscillations of the displacement jump, one can explain that the cohesive forces decrease gradually to zero. Therefore, by this effect of accumulation under cyclic loading, all the liaisons will finally break everywhere along the crack path, even if the amplitude of the loading is small. To our knowledge, this idea was first introduced by [23] in the context of Damage Mechanics. It is now a well-established principle which is included in all cohesive models used in fatigue. This concept was used again in $[18,19]$ and coupled with a variational approach consisting in a sequence of energy minimization problems. With these three fundamental ingredients: cohesive forces, accumulation of dissipated energy and energy minimization, it becomes possible to develop a general theory of crack propagation under any type of loading, monotonic as well as cyclic. Then, a particularly exciting challenge is to establish a link between the two paradoxical propagation laws of Griffith [17] and Paris [30, 31]. The former is generally considered as valid under monotonic loading, but must be replaced by the latter under cyclic loading. However, the latter has only a phenomenological character and is not based on well established physical foundations. Therefore, the challenge is to show that "good" cohesive models (in the sense above) give rise to Griffith-like crack 
propagation under monotonic loading and to Paris-like crack propagation under cyclic loading. This task was achieved in [19], but only in the one-dimensional simplified setting of a thin film peeling. That needs to introduce, as a last ingredient, an asymptotic method based on the presence of a small parameter. Indeed, a cohesive model necessarily contains (at least) one material characteristic length. For example, in Dugdale's model if $G_{c}$ denotes the usual critical energy release rate and $\sigma_{c}$ is the yield stress, then their ratio $d_{c}=G_{c} / \sigma_{c}$ is a characteristic length. If this length is small in comparison to the size of the body (and it is generally the case in the engineering structures), then it is possible (and even highly recommended) to analyze the asymptotic behavior of the response when the small parameter goes to zero. In the above mentioned simplified context, it was proved in [19] that the response governed by a cohesive model under monotonic loading (or small number of cycles) converges to that governed by Griffith's law, while the response under a large number of cycles converges to that of a Paris-like fatigue law. The construction of this "limit" fatigue law is even explicit in the case of Dugdale's model with a condition of irreversibility based on the concept of cumulated opening. In fact the limit fatigue law itself allows to establish the link between Griffith and Paris. Indeed, this law reads as $d \ell / d N=$ $f(G)$ where $\ell$ is the length of the crack, $N$ the number of cycles and $G$ is the energy release rate. The function $f$ is defined only for $G \leq G_{c}$, is undetermined when $G=G_{c}$, is monotonically increasing when $0<G<G_{c}$ and behaves like $C G^{m}$ when $G$ is small in comparison with $G_{c}$. In the case of a thin film, the exponent $m$ depends on the form of the strain energy. In any case, this elementary example shows that the challenge is reasonable.

The goal of the present paper is to extend this construction and the results to a more realistic two-dimensional setting. In fact, the results have been already announced in [1] and [2] but without any proof. All the construction and proofs are detailed here. Specifically, the paper is organized as follows. In Sect. 2, we introduce the cohesive model with the cumulated opening as the unique memory variable, the crack being assumed to be always in mode I. Then, we formulate the evolution problem from a variational approach in the general context of a two-dimensional body with a crack propagating along a predefined path. The two main ingredients of the variational formulation are a stability criterion and an energy balance. We obtain thus in a rational manner all the conditions that the normal stress, the opening, the opening rate and the cumulated opening must satisfy along the crack path. These conditions are particularized in the case of Dugdale's model and cyclic loading. Section 3 is the central one where the construction of the limit fatigue law is developed. Assuming that the characteristic length $d_{c}$ is small, we propose a two-scale method based on a priori assumptions which will be checked a posteriori. One of the main assumption is the concept of stationary regime at small scale which constitutes the cornerstone of the construction. Then, we assemble, step by step, the different components of the fatigue law. We distinguish in particular a large scale problem where the cohesive forces can be neglected and a small scale problem where they play an essential role. The former problem is structural by nature and hence will change from one problem to the other. On the other hand, the small scale problem is only dependent on the material behavior, the cohesive model and the type of cyclic loading, but not on the overall geometry and boundary conditions. In that sense, it has a universal character. The main difficulty of the paper is to solve this local problem (in a quasi closed form). That needs to determine the evolution along one cycle under the constraint imposed by the stationary regime assumption. One step in this procedure consists in solving a non linear equation involving $d \ell / d N$ and $G$. That equation comes from the cumulated opening rule, has the same form as in the case of the peeling test and enjoys the same properties. That yields the desired fatigue law of Paris-type $d \ell l d N=$ $f(G)$ which can be approximated by a power law $d \ell l d N=$ $C G^{2}$ when $G / G_{c}$ is small. The section ends by checking all the a priori assumptions. In Sect. 4 we establish some additional properties of the fatigue law. We study also the influence of the different "parameters" of the modeling on the derived fatigue law. We consider in particular the case when the unloading is not complete at the end of a cycle and establish a fatigue law in terms of the maximal value and the amplitude of the stress intensity factor, $d \ell / d N=$ $f\left(K^{M}, \Delta K\right)$. We compare also the fatigue law in mode I with that in mode III. The paper is completed by an appendix where a generic small scale problem is solved and which is used several times in Sect. 3 .

Let us complete this introduction by a short state-of-theart. There exists some attempts to recover fatigue laws from more fundamental mechanisms of propagation of defects, like [35] based on the dislocation theory, but they cannot be considered as really complete and satisfactory. The need for introducing cohesive forces so that to remove some fundamental drawbacks of Griffith's theory (like stress singularity or unphysical opening shape) is well known from the pioneering works by Dugdale [11] or Barenblatt [4]. A lot of interfacial models have been developed in this spirit, [27] being the prototype. The introduction of an irreversibility condition based on the concept of a loading-unloading hysteretic behavior like that proposed first in [23] is more recent, but tends to become the rule, see for instance $[3,22,28,32,33,36]$. However, these models are generally used in purely numerical studies with the objective to identify from computational tests the 
"effective" fatigue law. To our knowledge, there exists none work before [19] where a rigorous link is established between Griffith' law, Paris law and cohesive models. The reason is probably the lack, in all these computational works, of a theoretical framework in which it becomes possible to develop asymptotic methods. The variational approach provides this theoretical framework. Strongly inspired by the French School of Generalized Standard Materials and the works by NGuyen [29], it can be applied in a general setting of rate independent behaviors, see $[15,25]$. In the fracture mechanics setting, the variational approach has been largely developed since the late of nineteenths and the Francfort-Marigo paper [14]. Several theoretical results have been obtained in the Griffith theory setting, see [5, 9, 10]. Many improvements have been incorporated in order to include cohesive forces, see [5, 7, $12,16,20,24]$. But to our knowledge, the formulation of such cohesive models in the framework of a variational approach is due to [18]. Based on a "discrete in time" minimization problem, its "continuous in time" version has been proposed by [13], see also [5]. As far as a rigorous deduction of Paris fatigue law from cohesive models is concerned, we have knowledge of none work except $[1,2,18,19]$.

Throughout the paper we use classical notations: vectors and second order tensors are in bold face, their components are in italic, the inner product between vectors or tensors is indicated by a dot, like $\mathbf{f} \cdot \mathbf{u}$, time derivative by a dot, like $\dot{\mathbf{u}}$. No use is made of summation convention over repeated indices, $a^{+}$denotes the positive part of $a$, i.e., $a^{+}=\max \{a, 0\}$. The jump of a discontinuous field across a curve is denoted by double brackets, like $\llbracket u_{n} \rrbracket$. The dependence of a field (or more generally of any physical quantity) on a parameter is emphasized either by inserting the parameter inside square brackets just after the symbol of the field or by putting the parameter as a superscript. Thus, the displacement field at time $t$ is denoted by $\mathbf{u}^{t}$ and to emphasize its dependence on the current crack length $\ell$ it will be denoted $\mathbf{u}[\ell]^{t}$ (or simply $\mathbf{u}[\ell]$ if the value of $t$ is clear in context). From the technical standpoint, we essentially use the basic tools of the Calculus of Variations, some classical results of the theory of complex potentials [26] and basic concepts of Fracture Mechanics [6, 8, 21, 34].

\section{The cohesive model}

Throughout the paper, all the analysis is made in a twodimensional setting of plane strain. One uses a cartesian coordinate system $\left(x_{1}, x_{2}\right)$ with its canonical orthonormal basis $\left(\mathbf{e}_{1}, \mathbf{e}_{2}\right)$ and $\mathbf{e}_{3}=\mathbf{e}_{1} \wedge \mathbf{e}_{2}$ denotes the unit anti-plane vector. We consider a body, the reference configuration of which is the open subset $\Omega$ of $\mathbb{R}^{2}$, submitted to a time-dependent loading consisting in prescribed displacement $\xi^{t}$ on the part $\partial_{D} \Omega$ of the boundary, in prescribed surface forces $\mathbf{F}^{t}$ on the complementary part $\partial_{F} \Omega$ of the boundary and in prescribed body forces $\mathbf{f}^{t}$ in $\Omega$. All these data are supposed smooth, both in space and time. The loading causes the propagation of a crack along a predefined crack path $\hat{\Gamma}$, smooth simple curve with unit normal $\mathbf{n}$ and across which the displacement can be discontinuous. The simple curve representing the crack path $\hat{\Gamma}$ is parameterized by its arc length $s$, say $s \mapsto \hat{\mathbf{x}}(s)$. It will be often identified with the real interval $\left[0, L_{c}\right], L_{c}$ being its length. In the unbreakable part $\Omega \backslash \hat{\Gamma}$ of the body, the material has an isotropic linear elastic behavior characterized by Young's modulus $E$, Poisson's ratio $v$ and Lamé's coefficients $\lambda, \mu$.

We assume that the body, the loading and the crack path are so that the crack is always in mode I, i.e., only the normal displacement can be discontinuous on $\hat{\Gamma}$. We denote by $\llbracket u_{n}^{t} \rrbracket$ and $\llbracket \dot{u}_{n}^{t} \rrbracket$ the jump and the rate of the jump of the normal component of the displacement at time $t$ at a point on the crack path and call them the opening and the rate of opening. The opening must satisfy the non interpenetration condition $\llbracket u_{n}^{t} \rrbracket \geq 0$ at every $t$ :

$\llbracket \mathbf{u}^{t} \rrbracket=\llbracket u_{n}^{t} \rrbracket \mathbf{n}, \quad \llbracket u_{n}^{t} \rrbracket \geq 0, \quad$ on $\quad \hat{\Gamma}$.

Accordingly, "by symmetry", the tangential stress always vanishes on $\hat{\Gamma}$. We denote by $\sigma_{n n}^{t}$ the normal component of the stress vector and call it the cohesive force when it is positive and the contact force when it is negative. The relationship between the current cohesive force and the current opening (or, more generally, the history of the opening up to the current time) is obtained via a variational approach from fundamental assumptions on the surface energy density.

Remark 1 This mode I assumption could appear very strong as it is formulated above and only valid in very few cases. Indeed, the tangential displacement will be continuous through $\hat{\Gamma}$ only in the case of a symmetric body, a symmetric loading and a symmetric crack path. However, in order that the two-scale procedure developed in Sect. 3 remains valid it is sufficient that this condition holds in the neighborhood of the crack tip. Specifically, it is sufficient that $\mathrm{K}_{\mathrm{II}}[\ell]=0$ for all $\ell \in\left(0, L_{c}\right), \mathrm{K}_{\mathrm{II}}[\ell]$ being the mode II stress intensity factor appearing in the large scale problem of Sect. 3.2. Therefore, the condition is local in space at given $t$ and is satisfied for every $t$ if the crack path is such that the propagation follows the Local Symmetry Principle.

\subsection{The variational formulation without any irreversibility condition}

Let us first consider the case when the opening is always monotonically increasing in time, i.e., when $\llbracket u_{n}^{0} \rrbracket=0$ and $\llbracket \dot{u}_{n}^{t} \rrbracket \geq 0$. Then it is useless to introduce any condition of 
irreversibility and the surface energy density is a function $\phi$ of $\llbracket u_{n} \rrbracket$ alone. To obtain precise mathematical results, it is necessary to suppose that $\phi$ enjoys some relevant concave and monotonic properties, see [24]. However, since in this paper all the developments will be made with Dugdale's surface energy, we simply assume that $\phi$ is defined on $[0,+\infty)$, monotonically increasing and piecewise smooth with $\phi(0)=0$ and $\sigma_{c}=\phi^{\prime}(0)>0$, the prime denoting the derivative.

Since the response of the body depends on the current loading only and not on its history, we omit the superscript $t$. Let $\mathbf{v}$ be a kinematically admissible displacement (with the constraint that only its normal component can be discontinuous on $\hat{\Gamma})$, i.e.,

$$
\begin{aligned}
\mathbf{v} \in \mathcal{C}= & \left\{\mathbf{v} \in H^{1}\left(\Omega \backslash \Gamma, \mathbb{R}^{2}\right): \mathbf{v}=\xi\right. \\
& \text { on } \left.\partial_{D} \Omega, \llbracket \mathbf{v} \rrbracket=\llbracket v_{n} \rrbracket \mathbf{n} \quad \text { with } \llbracket v_{n} \rrbracket \geq 0 \text { on } \hat{\Gamma}\right\}
\end{aligned}
$$

where $H^{1}$ denotes the usual Sobolev space. The associated total energy of the body is given by

$$
\begin{aligned}
\mathcal{E}(\mathbf{v})= & \int_{\Omega \backslash \hat{\Gamma}} \frac{1}{2} \mathrm{~A} \boldsymbol{\varepsilon}(\mathbf{v}) \cdot \boldsymbol{\varepsilon}(\mathbf{v}) d x+\int_{\hat{\Gamma}} \phi\left(\llbracket v_{n} \rrbracket\right) d s-\int_{\Omega} \mathbf{f} \cdot \mathbf{v} d x \\
& -\int_{\partial_{F} \Omega} \mathbf{F} \cdot \mathbf{v} d s
\end{aligned}
$$

where A denotes the stiffness tensor of the material and $\varepsilon(\mathbf{v})$ is the symmetric part of the gradient of $\mathbf{v}$. The true displacement field $\mathbf{u}$ is (the) one in $\mathcal{C}$ which satisfies the following local minimality condition:

$\forall \mathbf{v} \in \mathcal{C}, \quad \exists \bar{h}>0: \forall h \in[0, \bar{h}], \quad \mathcal{E}(\mathbf{u}) \leq \mathcal{E}(\mathbf{u}+h(\mathbf{v}-\mathbf{u}))$.

Dividing by $h>0$ the inequality above and passing to the limit when $h \downarrow 0$, we obtain the so-called first order optimality condition

$$
\begin{aligned}
\forall \mathbf{v} & \in \mathcal{C}, \quad \int_{\Omega \backslash \hat{\Gamma}} \boldsymbol{\sigma} \cdot \boldsymbol{\varepsilon}(\mathbf{v}-\mathbf{u}) d x+\int_{\hat{\Gamma}} \phi^{\prime}\left(\llbracket u_{n} \rrbracket\right) \llbracket v_{n}-u_{n} \rrbracket d s \\
& \geq \int_{\Omega} \mathbf{f} \cdot(\mathbf{v}-\mathbf{u}) d x+\int_{\partial_{F} \Omega} \mathbf{F} \cdot(\mathbf{v}-\mathbf{u}) d s
\end{aligned}
$$

where $\sigma=A \varepsilon(\mathbf{u})$ denotes the stress field. The variational inequality (2) is equivalent to a system of local equalities and inequalities which are obtained by considering different types of test fields $\mathbf{v}$.

1. Let $\mathbf{v}=\mathbf{u}+\mathbf{w}$ with $\mathbf{w}=\mathbf{0}$ on $\partial_{D} \Omega$ and $\llbracket w_{n} \rrbracket=0$ on $\hat{\Gamma}$. Inserting into (2) and using standard arguments of Calculus of Variations lead to the local equilibrium equations and the natural boundary conditions

$$
\operatorname{div} \boldsymbol{\sigma}+\mathbf{f}=\mathbf{0} \text { in } \Omega \backslash \hat{\Gamma}, \quad \boldsymbol{\sigma} \mathbf{n}=\mathbf{F} \quad \text { on } \partial_{F} \boldsymbol{\Omega} .
$$

Moreover, we obtain also that the normal stress is continuous on $\hat{\Gamma}$ while we assume that "by symmetry" the shear stress vanishes on $\hat{\Gamma}$ :

$\boldsymbol{\sigma} \mathbf{n}=\sigma_{n n} \mathbf{n}, \quad \llbracket \sigma_{n n} \rrbracket=0 \quad$ on $\hat{\Gamma}$.

After inserting (3) and (4) into (2), the first order optimal condition becomes

$\forall \mathbf{v} \in \mathcal{C}, \quad \int_{\hat{\Gamma}}\left(\phi^{\prime}\left(\llbracket u_{n} \rrbracket\right)-\sigma_{n n}\right) \llbracket v_{n}-u_{n} \rrbracket d s \geq 0$.

2. Let us divide $\hat{\Gamma}$ into two parts: $\Gamma_{\mathrm{c}}$ where $\llbracket u_{n} \rrbracket=0$ and $\Gamma_{\circ}$ where $\llbracket u_{n} \rrbracket>0$. We obtain that (5) is satisfied if and only if the cohesive forces repartition verifies

$$
\left\{\begin{array}{lll}
\sigma_{n n} \leq \sigma_{c}=\phi^{\prime}(0) & \text { on } & \Gamma_{c}=\left\{s \in \hat{\Gamma}: \llbracket\left[u_{n} \rrbracket\right](s)=0\right\} \\
\sigma_{n n}=\phi^{\prime}\left(\llbracket u_{n} \rrbracket\right) & \text { on } & \Gamma_{\circ}=\left\{s \in \hat{\Gamma}: \llbracket u_{n} \rrbracket(s)>0\right\}
\end{array} .\right.
$$

Remark 2 The first order optimality condition (2) contains not only the normal stress-opening relation $\sigma_{n n}=$ $\phi^{\prime}\left(\left[\left[u_{n}\right]\right]\right)$ but also the stress yield criterion $\sigma_{n n} \leq \sigma_{c}$ for the onset of opening. In the present case where only the normal displacement is discontinuous, this criterion is simply a maximal traction criterion. This fundamental result can be generalized in a three dimensional setting for general surface energy densities to obtain more general stress yield criteria, see [20] and [7].

\subsection{Introduction of an irreversibility condition and the new evolution problem}

When the surface energy density depends only on the current opening, the response of the body depends only on the current loading. Therefore, in the case of cyclic loading, the response is the same at each cycle, the crack cannot propagate progressively from one cycle to the other and no fatigue effect is possible. Accordingly, we must introduce irreversibility conditions and consider that the surface energy density depends on the entire history of the opening. To this end, the normal stress-opening relation is customarily defined by using memory variables, say $\delta$, see $[22,28$, $32,33,36]$. In a variational approach that consists first in defining the surface energy density as a function of the current opening and these memory variables. Here, in a two-dimensional mode I setting, we make the simplest choice as in $[2,18,19]$. We choose as memory variable the cumulated opening up to time $t$ defined at each point $s$ of the crack path by

$\delta^{t}(s)=\delta^{0}(s)+\int_{0}^{t}\left(\llbracket \dot{u}_{n}^{t} \rrbracket(s)\right)^{+} d t^{\prime}$ 
where $\delta^{0}$ represents the given initial value of the cumulated opening and the + denotes the positive part. In a differential form, (7) reads as

$\dot{\delta}=\llbracket \dot{u}_{n} \rrbracket^{+}$.

Then, we assume that the surface energy density is the same function $\phi$ as in the previous subsection, but now depending on the cumulated opening instead of the opening. Accordingly, the surface energy of the body at time $t$, say $\mathcal{S}^{t}$, reads as

$\mathcal{S}^{t}=\int_{\hat{\Gamma}} \phi\left(\delta^{t}(s)\right) d s$.

In the spirit of the ideas developed in [5, 13, 25], the evolution of the cracking in the body is defined in terms of a stability criterion and an energy balance principle.

Let us first introduce the stability condition. To test the stability of the state $\left(\mathbf{u}^{t}, \delta^{t}\right)$ of the body at time $t \geq 0$, we consider a kinematically admissible displacement $\mathbf{v}$ at time $t$, i.e., $\mathbf{v} \in \mathcal{C}^{t}$ with

$\mathcal{C}^{t}=\left\{\mathbf{v} \in H^{1}\left(\Omega \backslash \hat{\Gamma}, \mathbb{R}^{2}\right): \mathbf{v}=\xi^{t}\right.$ on $\partial_{D} \Omega$,

$\llbracket \mathbf{v} \rrbracket=\llbracket v_{n} \rrbracket \mathbf{n}$ with $\llbracket v_{n} \rrbracket \geq 0$ on $\left.\hat{\Gamma}\right\}$.

Replacing the true displacement $\mathbf{u}^{t}$ by the virtual one $\mathbf{v}$, the deformation of the body undergoes the virtual jump discontinuity $\mathbf{v}-\mathbf{u}^{t}$ at time $t$ and therefore the associated virtual cumulated opening at time $t$ reads as $\delta_{*}^{t}=\delta^{t}+$ $\llbracket v_{n}-u_{n}^{t} \rrbracket^{+}$. Accordingly, the total energy of the body at time $t$ associated with this virtual displacement $\mathbf{v}$ reads as

$$
\begin{aligned}
\mathcal{E}^{t}(\mathbf{v})= & \int_{\Omega \backslash \hat{\Gamma}} \frac{1}{2} \mathbf{A} \boldsymbol{\varepsilon}(\mathbf{v}) \cdot \boldsymbol{\varepsilon}(\mathbf{v}) d x+\int_{\hat{\Gamma}} \phi\left(\delta^{t}+\llbracket v_{n}-u_{n}^{t} \rrbracket^{+}\right) d s \\
& -\int_{\Omega} \mathbf{f}^{t} \cdot \mathbf{v} d x-\int_{\partial_{F} \Omega} \mathbf{F}^{t} \cdot \mathbf{v} d s .
\end{aligned}
$$

The stability condition consists in using the local minimality condition (1) with the new expression of the energy, i.e.,

$$
\begin{gathered}
\forall \mathbf{v} \in \mathcal{C}^{t}, \quad \exists \bar{h}>0: \forall h \in[0, \bar{h}], \\
\mathcal{E}^{t}\left(\mathbf{u}^{t}\right) \leq \mathcal{E}^{t}\left(\mathbf{u}^{t}+h\left(\mathbf{v}-\mathbf{u}^{t}\right)\right) .
\end{gathered}
$$

However, the stability condition alone is not sufficient to define the evolution of the body. It must be completed by the energy balance which reads as

$$
\begin{aligned}
& \mathcal{E}^{t}\left(\mathbf{u}^{t}\right)=\mathcal{E}^{0}\left(\mathbf{u}^{0}\right) \\
& +\int_{0}^{t}\left(\int_{\partial_{D} \Omega} \boldsymbol{\sigma}^{t^{\prime}} \mathbf{n} \cdot \dot{\boldsymbol{\xi}}^{t^{\prime}} d s-\int_{\Omega} \dot{\mathbf{f}}^{t^{\prime}} \cdot \mathbf{u}^{t^{\prime}} d x-\int_{\partial_{F} \Omega} \dot{\mathbf{F}}^{t^{\prime}} \cdot \mathbf{u}^{t^{\prime}} d s\right) d t^{\prime}
\end{aligned}
$$

where $\dot{\mathbf{f}}, \dot{\mathbf{F}}$ and $\dot{\xi}$ denote the rate of the data. In (10), $\mathbf{u}^{0}$ denotes the initial displacement field which must be compatible with the stability condition (9) written at $t=0$ with the data $\delta^{0}, \mathbf{f}^{0}, \mathbf{F}^{0}$ and $\xi^{0}$.

Remark 3 Therefore, the evolution problem consists in finding $t \mapsto\left(\mathbf{u}^{t}, \delta^{t}\right)$ which satisfies, at every $t \geq 0$, (7), (9) and (10). The main advantages of such a formulation are the following ones:

1. it is valid for any type of loading (with the unique restriction that the loading is smooth in time) and can be used both for monotone or cyclic loading;

2. it can be easily extended to a very general framework (3D, anisotropic and heterogeneous body, ...);

3. it contains a stability condition which can be used as a criterion of selection of solutions. Indeed, since the surface energy density is usually a concave function of $\delta$, the total energy is not a convex function of $\mathbf{v}$ and the uniqueness of the solution is not guaranteed;

4. it does not require that the evolution is smooth in time (only the data have to be smooth). It allows to search for discontinuous-in-time solutions.

Note that the proposed evolution law is rate independent. Indeed, let $t \mapsto \mathbf{u}^{t}$ be a solution of (9) and (10) with the data $\delta^{0}$ and $t \mapsto\left(\mathbf{f}^{t}, \mathbf{F}^{t}, \boldsymbol{\xi}^{t}\right)$. If a change of the rate of loading is made by considering a smooth one-to-one map $t \mapsto \tau(t)$ such that $\tau(0)=0$ and $d \tau / d t>0$, then $t \mapsto \tilde{\mathbf{u}}^{t}=\mathbf{u}^{\tau(t)}$ is a solution of the problem with the new data $t \mapsto\left(\mathbf{f}^{\tau(t)}, \mathbf{F}^{\tau(t)}, \boldsymbol{\xi}^{\tau(t)}\right)$.

From now on, we assume that there is no initial cumulated opening, $\delta^{0}=0$ on $\hat{\Gamma}$, the loading starts from 0 , i.e. $\mathbf{f}^{0}=\mathbf{F}^{0}=\boldsymbol{\xi}^{0}=\mathbf{0}$, so that $\mathbf{u}^{0}=\mathbf{0}$. Let us assume that the evolution problem has a smooth solution and let us proceed as in the previous subsection to obtain the local conditions satisfied by this solution (in particular, the normal stressopening relations).

Let $t>0$, dividing (9) by $h>0$ and passing to the limit as $h \downarrow 0$, we obtain the first order stability condition

$$
\begin{aligned}
\forall \mathbf{v} & \in \mathcal{C}^{t}, \quad \int_{\Omega \backslash \hat{\Gamma}} \boldsymbol{\sigma}^{t} \cdot \boldsymbol{\varepsilon}\left(\mathbf{v}-\mathbf{u}^{t}\right) d x+\int_{\hat{\Gamma}} \phi^{\prime}\left(\delta^{t}\right) \llbracket v_{n}-u_{n}^{t} \rrbracket^{+} d s \\
& \geq \int_{\Omega} \mathbf{f}^{t} \cdot\left(\mathbf{v}-\mathbf{u}^{t}\right) d x+\int_{\partial_{F} \Omega} \mathbf{F}^{t} \cdot\left(\mathbf{v}-\mathbf{u}^{t}\right) d s
\end{aligned}
$$

Let us first take $\mathbf{v}=\mathbf{u}^{t}+\mathbf{w}$ with $\mathbf{w}=\mathbf{0}$ on $\partial_{D} \Omega$ and $\llbracket w_{n} \rrbracket=0$ on $\hat{\Gamma}$. Inserting into (11) gives, as in the previous subsection, the equilibrium equations and the natural boundary conditions,

$\operatorname{div} \boldsymbol{\sigma}^{t}+\mathbf{f}^{t}=\mathbf{0} \quad$ in $\Omega \backslash \hat{\Gamma}, \quad \boldsymbol{\sigma}^{t} \mathbf{n}=\mathbf{F}^{t} \quad$ on $\partial_{F} \Omega$,

as well as the continuity of the normal stress on $\hat{\Gamma}$ (and we still assume the vanishing of the shear stress), 
$\boldsymbol{\sigma}^{t} \mathbf{n}=\sigma_{n n}^{t} \mathbf{n}, \quad \llbracket \sigma_{n n}^{t} \rrbracket=0 \quad$ on $\hat{\Gamma}$.

After inserting (12) and (13) into (11), the first order stability condition becomes

$\forall \mathbf{v} \in \mathcal{C}^{t}, \quad \int_{\hat{\Gamma}}\left(\phi^{\prime}\left(\delta^{t}\right) \llbracket v_{n}-u_{n}^{t} \rrbracket^{+}-\sigma_{n n}^{t} \llbracket v_{n}-u_{n}^{t} \rrbracket\right) d s \geq 0$.

Let us divide $\hat{\Gamma}$ into two parts defined in terms of $\llbracket u_{n}^{t} \rrbracket: \Gamma_{\mathrm{c}}^{t}$ (c like closed) where $\llbracket u_{n}^{t} \rrbracket=0$ and $\Gamma_{\text {o }}^{t}$ (o like open) where $\llbracket u_{n}^{t} \rrbracket>0$. We obtain that (14) is satisfied if and only if the cohesive forces repartition is such that

$$
\left\{\begin{array}{lll}
\sigma_{n n}^{t} \leq \phi^{\prime}\left(\delta^{t}\right) & \text { on } & \Gamma_{\mathrm{c}}^{t}=\left\{s \in \hat{\Gamma}: \llbracket u_{n}^{t} \rrbracket(s)=0\right\} \\
\sigma_{n n}^{t} \in\left[0, \phi^{\prime}\left(\delta^{t}\right)\right] & \text { on } & \Gamma_{\mathrm{o}}^{t}=\left\{s \in \hat{\Gamma}: \llbracket u_{n}^{t} \rrbracket(s)>0\right\}
\end{array}\right. \text {. }
$$

We see that, because of the introduction of an irreversibility condition (through the concept of cumulated opening), the first order stability condition is no more sufficient to obtain all the information concerning the cohesive forces. We have also to consider the energy balance. Assuming that the evolution is smooth, differentiating (10) with respect to time and using (8) with $\mathbf{v}=\mathbf{u}^{t}$, we get

$$
\begin{aligned}
& \int_{\Omega \backslash \hat{\Gamma}} \boldsymbol{\sigma}^{t} \cdot \boldsymbol{\varepsilon}\left(\dot{\mathbf{u}}^{t}\right) d x+\int_{\hat{\Gamma}} \phi^{\prime}\left(\delta^{t}\right) \dot{\delta}^{t} d s \\
& \quad=\int_{\Omega} \mathbf{f}^{t} \cdot \dot{\mathbf{u}}^{t} d x+\int_{\partial_{F} \Omega} \mathbf{F}^{t} \cdot \dot{\mathbf{u}}^{t} d s+\int_{\partial_{D} \Omega} \boldsymbol{\sigma}^{t} \mathbf{n} \cdot \dot{\boldsymbol{\xi}}^{t} d s .
\end{aligned}
$$

Integrating by parts the first integral in the left hand side of (16) and using (12)-(13) we obtain

$$
\int_{\hat{\Gamma}}\left(\phi^{\prime}\left(\delta^{t}\right) \llbracket \dot{u}_{n}^{t} \rrbracket^{+}-\sigma_{n n}^{t} \llbracket \dot{u}_{n}^{t} \rrbracket\right) d s=0 .
$$

After dividing $\hat{\Gamma}$ into three parts defined in terms of $\llbracket \dot{u}_{n}^{t} \rrbracket: \Gamma_{\mathrm{a}}^{t}(a$ like active $)$ where $\llbracket \dot{u}_{n}^{t} \rrbracket>0, \Gamma_{\mathrm{n}}^{t}(n$ like neutral $)$ where $\llbracket \dot{u}_{n}^{t} \rrbracket=0$ and $\Gamma_{\mathrm{p}}^{t}(p$ like passive $)$ where $\llbracket \dot{u}_{n}^{t} \rrbracket<0$, (17) becomes

$$
\int_{\Gamma_{\mathrm{a}}^{t}}\left(\phi^{\prime}\left(\delta^{t}\right)-\sigma_{n n}^{t}\right) \llbracket \dot{u}_{n}^{t} \rrbracket d s+\int_{\Gamma_{\mathrm{p}}^{t}} \sigma_{n n}^{t}\left|\llbracket \dot{u}_{n}^{t} \rrbracket\right| d s=0 .
$$

By virtue of (15), the first integral of (18) is non negative and vanishes if and only if $\sigma_{n n}^{t}=\phi^{\prime}\left(\delta^{t}\right)$ on $\Gamma_{\mathrm{a}}^{t}$. Because of the non interpenetration condition, $\Gamma_{\mathrm{p}}^{t} \subset \Gamma_{\mathrm{o}}^{t}$ and hence, by virtue of (15), the second integral of (18) is also non negative and vanishes if and only if $\sigma_{n n}^{t}=0$ on $\Gamma_{\mathrm{p}}^{t}$. Consequently, both integrals must vanish and we have finally obtained the following normal stress-opening relations which complete those of (15) (which have still to be satisfied):

$$
\left\{\begin{array}{lll}
\sigma_{n n}^{t}=\phi^{\prime}\left(\delta^{t}\right) & \text { on } & \Gamma_{\mathrm{a}}^{t}=\left\{s \in \hat{\Gamma}: \llbracket \dot{u}_{n}^{t} \rrbracket(s)>0\right\} \\
\sigma_{n n}^{t} \leq \phi^{\prime}\left(\delta^{t}\right) & \text { on } & \Gamma_{\mathrm{n}}^{t}=\left\{s \in \hat{\Gamma}: \llbracket \dot{u}_{n}^{t} \rrbracket(s)=0\right\} \\
\sigma_{n n}^{t}=0 & \text { on } & \Gamma_{\mathrm{p}}^{t}=\left\{s \in \hat{\Gamma}: \llbracket \dot{u}_{n}^{t} \rrbracket(s)<0\right\}
\end{array} .\right.
$$

Remark 4 It appears that the cohesive force depends not only on the opening but also on the rate of opening. The cohesive force is activated when the opening increases but is deactivated when the opening decreases. It is not the value of the opening which is important, but the sign of its rate and the value of its cumulation. That constitutes the key property to obtain fatigue effects under cyclic loading. This idea was first introduced by [23] in the context of Damage Mechanics. Note that if the opening is always increasing once the crack is open, i.e., if $\llbracket \dot{u}_{n}^{t} \rrbracket>0$ when $\delta^{t}>0$, then $\delta^{t}=\llbracket u_{n}^{t} \rrbracket$ at every $t$ and by applying the conditions (15) and (19) we recover (6). In other words, under monotone loading the two formulations are equivalent. The irreversibility condition plays a role only under non monotonic loading.

The set of conditions (12), (13), (15) and (19) are only necessary conditions in order that the stability condition (9) be satisfied. They are, in general, not sufficient and second order stability conditions should be considered. However, we shall develop hereafter the construction of the fatigue law only from the first order stability conditions, the study of the role of the additional conditions is left for future work.

\subsection{Case of Dugdale's surface energy and of cyclic loading}

Let us particularize the previous formulation and results to the case of Dugdale's model and cyclic loading. In the case of Dugdale's model, the surface energy density is defined on $[0,+\infty)$ by

$\phi(\delta)= \begin{cases}G_{c} \frac{\delta}{d_{c}} & \text { if } 0 \leq \delta \leq d_{c} \\ G_{c} & \text { if } \delta \geq d_{c}\end{cases}$

where $G_{c}$ is the critical energy release rate of Griffith's theory and $d_{c}$ is a characteristic length of the material. Therefore the critical stress is $\sigma_{c}=G_{c} / d_{c}$. The main particularities of Dugdale's model are

1. The cohesive force vanishes as soon as the cumulated opening becomes larger than $d_{c}$. Accordingly, the crack path can be divided into three zones (which evolve with time): the still bonded zone $\Gamma_{\mathrm{B}}^{t}$ where $\delta^{t}=0$, the process zone (or genuine cohesive zone) $\Gamma_{\mathrm{C}}^{t}$ where $0<\delta^{t}<d_{c}$ and the already debonded zone (or non cohesive zone) $\Gamma_{\mathrm{D}}^{t}$ where $\delta^{t}>d_{c}$. (Because of the concavity of $\phi$ and its non differentiability at $\delta=d_{c}$, 
the points where $\delta^{t}=d_{c}$ are isolated and can be neglected in this partitioning.)

2. $\phi$ is piecewise linear and hence its derivative is piecewise constant. Consequently, the cohesive force is constant and equal to $\sigma_{c}$ on the active part of the process zone whereas it vanishes everywhere on $\Gamma_{\mathrm{D}}^{t}$. This property is very useful in the sequel to obtain solutions in a closed form.

The normal stress-opening conditions read now as

$$
\begin{aligned}
& \left\{\begin{array}{lll}
\sigma_{n n}^{t} \leq \sigma_{c} & \text { on } \quad \Gamma_{\mathrm{c}}^{t} \cap\left(\Gamma_{\mathrm{B}}^{t} \cup \Gamma_{\mathrm{C}}^{t}\right) \\
\sigma_{n n}^{t} \leq 0 & \text { on } & \Gamma_{\mathrm{c}}^{t} \cap \Gamma_{\mathrm{D}}^{t}
\end{array}\right. \\
& \left\{\begin{array}{lll}
\sigma_{n n}^{t}=\sigma_{c} & \text { on } \quad \Gamma_{\mathrm{Ca}}^{t} \\
\sigma_{n n}^{t} \in\left[0, \sigma_{c}\right] & \text { on } \quad \Gamma_{\mathrm{o}}^{t} \cap \Gamma_{\mathrm{Cn}}^{t} \\
\sigma_{n n}^{t}=0 & \text { on } \quad \Gamma_{\mathrm{o}}^{t} \cap\left(\Gamma_{\mathrm{Cp}}^{t} \cup \Gamma_{\mathrm{D}}^{t}\right)
\end{array}\right.
\end{aligned}
$$

with

$$
\begin{aligned}
& \left\{\begin{array}{l}
\Gamma_{\mathrm{B}}^{t}=\left\{\mathbf{x} \in \hat{\Gamma}: \delta^{t}(\mathbf{x})=0\right\} \\
\Gamma_{\mathrm{C}}^{t}=\left\{\mathbf{x} \in \hat{\Gamma}: 0<\delta^{t}(\mathbf{x})<d_{c}\right\}, \\
\Gamma_{\mathrm{D}}^{t}=\left\{\mathbf{x} \in \hat{\Gamma}: \delta^{t}(\mathbf{x})>d_{c}\right\}
\end{array}\right. \\
& \left\{\begin{array}{c}
\Gamma_{\mathrm{Ca}}^{t}=\left\{\mathbf{x} \in \Gamma_{\mathrm{C}}^{t}: \llbracket \dot{u}_{n}^{t} \rrbracket(\mathbf{x})>0\right\} \\
\Gamma_{\mathrm{Cn}}^{t}=\left\{\mathbf{x} \in \Gamma_{\mathrm{C}}^{t}: \llbracket \dot{u}_{n}^{t} \rrbracket(\mathbf{x})=0\right\} . \\
\Gamma_{\mathrm{Cp}}^{t}=\left\{\mathbf{x} \in \Gamma_{\mathrm{C}}^{t}: \llbracket \dot{u}_{n}^{t} \rrbracket(\mathbf{x})<0\right\}
\end{array}\right.
\end{aligned}
$$

Only the cohesive zone has to be divided into active, neutral and passive zones, since the cohesive forces are equal to 0 in the debonded zone forever (but contact forces can be present). We consider a particular type of cyclic loading, called simple cyclic loading and defined as follows. The loading is proportional in the sense that

$\mathbf{f}^{t}=\varpi(t) q_{M} \overline{\mathbf{f}}, \quad \mathbf{F}^{t}=\varpi(t) q_{M} \overline{\mathbf{F}}, \quad \xi^{t}=\varpi(t) q_{M} \bar{\xi}$

where $\overline{\mathbf{f}}, \overline{\mathbf{F}}$ and $\bar{\xi}$ are normalized data which do not depend on time and $q_{M}$ is the amplitude of the loading. Furthermore, the real-valued function $\varpi$ is a seesaw-type function, i.e., periodic with period 2 and defined on $[0,2]$ by

$\varpi(t)= \begin{cases}t & \text { if } 0 \leq t \leq 1 \\ (2-t) & \text { if } 1 \leq t \leq 2 .\end{cases}$

By virtue of the rate-independent character of the evolution law, only the monotonic properties of $\varpi$ are important, its piecewise linear character and the value of the period have no influence. For $i \in \mathbb{N}_{*}$, we call cycle $i$ or $i$ th cycle the time interval $[2(i-1), 2 i)$, loading phase of cycle $i$ the time interval $(2(i-1), 2 i-1)$, unloading phase of cycle $i$ the time interval $(2 i-1,2 i)$, end of loading of cycle $i$ the time $t=2 i-1$ and end of unloading of cycle $i$ the time $t=2 i$.

\section{Construction of the fatigue law}

3.1 Main a priori assumptions and the two-scale procedure

Throughout the section we consider only Dugdale's cohesive model with a simple cyclic loading. Moreover, we suppose that the size of the body is large in comparison with the internal length of the material and hence that the ratio $\varepsilon=d_{c} / L_{c}$ is a small dimensionless parameter:

$\epsilon=\frac{d_{c}}{L_{c}} \ll 1$.

Therefore, $G_{c}=\sigma_{c} d_{c}=\varepsilon \sigma_{c} L_{c}$. The surface energy necessary to debond all the crack path is equal to $\epsilon \sigma_{c} L_{c}^{2}$ and hence of the order of $\varepsilon$. We assume that the potential energy is also of the order of $\varepsilon$ what requires that the amplitude of the loading is of the order of $\sqrt{\epsilon}$ (otherwise, if the order of the loading amplitude is smaller than $\sqrt{\epsilon}$, then the crack will not propagate, while, if the order of the loading amplitude is larger than $\sqrt{\epsilon}$, then all the crack path will debond during the first loading phase). Accordingly, we set

$q_{M}=\sqrt{\epsilon}$.

But even in this restricted context, the properties of the solution (if any) of the evolution problem strongly depend on the geometry of the body, on the crack path and on the data. It is not possible to follow a purely deductive procedure and to obtain precise results without particularizing the problem, as in [19]. Therefore, we shall proceed as follows:

1. We make a priori assumptions on the form of the solution, in particular on the shape and the size of the different zones of the crack path;

2. We develop a two-scale approach based on the smallness of the internal length $d_{c}$;

3. We check a posteriori the pertinence of the a priori assumptions.

Hypothesis. The first a priori assumptions are the following ones (additional assumptions will be introduced later):

H1 At time $t$, the non cohesive zone is the interval $\Gamma_{\mathrm{D}}^{t}=\left[0, \ell^{t}\right)$, the process zone is the interval $\Gamma_{\mathrm{C}}^{t}=$ $\left(\ell^{t}, \ell^{t}+\mathrm{d}^{t}\right)$ and the bonded zone is the interval $\Gamma_{\mathrm{B}}^{t}=\left[\ell^{t}+\mathrm{d}^{t}, L_{c}\right]$;

H2 The length $\mathrm{d}^{t}$ of the process zone is of the order of $d_{c}$ and hence small in comparison with $L_{c}$;

H3 During any cycle $i$, the propagation of the tip of the non cohesive zone, that is $\ell^{2 i}-\ell^{2 i-2}$, is of the order of $d_{c}$ and hence small in comparison with $L_{c}$;

H4 From one cycle to the other, at first approximation, the evolution follows a quasi-stationary regime. 
The first assumption is only introduced to simplify the presentation, the case with several process zones and several non cohesive crack tips could be treated in the same manner. The meaning of the last assumption will become precise later. The second and the third assumptions allow a scale separation, both in space and time.

Let $\ell$ be given in the interval $\left(0, L_{c}\right)$, independent of $\varepsilon$. From H3, we deduce that a great number of cycles are necessary, say $N_{\varepsilon}$, so that the length of the non cohesive crack be equal to $\ell . N_{\varepsilon}$ is of the order of $1 / \varepsilon$ and if we consider $T=\lim _{\varepsilon \rightarrow 0} \varepsilon N_{\varepsilon}, T$ can be seen as the real valued parameter characterizing the number of cycles at the macro-scale. The main goal of the subsequent analysis is to find the relation between $T$ and $\ell$, that is the function $T \mapsto \ell(T)$ giving the evolution of the tip of the non cohesive crack at the macro-scale number of cycles. That requires to also consider the evolution problem at a small scale. Specifically, for a given $i \in \mathbb{Z}$, independent of $\varepsilon$, if we consider the cycle $N_{\varepsilon}+i$ or equivalently $T / \varepsilon+i$, then $i$ can be seen as the micro-scale number of cycles. By Hypothesis $\mathbf{H 3}$ again, the propagation of the non cohesive crack tip during the cycle $N_{\varepsilon}+i$ is of the order of $\varepsilon$ and a priori $\lim _{\epsilon \rightarrow 0}\left(\ell^{2 N_{\epsilon}+2 i}-\ell^{2 N_{\epsilon}-2 i-2}\right) / \epsilon$ depends on $T$ (or $\ell$ ) and $i$. But one main feature of Hypothesis $\mathbf{H 4}$ is to claim that this limit is independent of $i$, say $\dot{\ell}(T)$. Accordingly, $\ell^{2 N_{\epsilon}+2 i}=\ell(T)+\epsilon i \dot{\ell}(T)+o(\epsilon)$ and $\dot{\ell}(T)$ can be identified with $\frac{d \ell}{d T}(T)$. The main step in order to determine $T \mapsto \ell(T)$ consists in finding the relation between $\dot{\ell}(T)$ and global energetic quantities characterizing the state of the cracked body at "time" $T$. For that, a separation of scale in space is also necessary. Specifically, we shall first construct an approximation of the displacement field at a large scale, i.e., at the scale of the whole body, with the help of Assumptions H1-H3. This so-called large scale problem will give us the macroscopic energetic quantities like the effective Stress Intensity Factor $\mathrm{K}_{\mathrm{I}}$ in terms of the length $\ell$ of the non cohesive crack. Then we shall make a zoom of the tip of the non cohesive crack at the macro-time $T$ and consider the evolution problem at a micro-scale both in space and time. This so-called small scale problem will give the relation between $\dot{\ell}$ and $\mathrm{K}_{\mathrm{I}}$.

\subsection{The large scale problem}

At time $t=2 N_{\varepsilon}-1$, i.e., at the end of the loading phase of the cycle $N_{\varepsilon}$, the amplitude of the loading is $\sqrt{\epsilon}$, the tip of the non cohesive crack is at $\ell$ and the length of the process zone is of the order of $\varepsilon$. At a large scale, if we neglect the process zone, the true displacement and stress fields $\left(\mathbf{u}^{2 N_{\epsilon}-1}, \boldsymbol{\sigma}^{2 N_{\epsilon}-1}\right)$ can be approximated by $(\sqrt{\epsilon} \mathbf{u}[\ell], \sqrt{\epsilon} \boldsymbol{\sigma}[\ell])$ (to avoid any confusion, the dependence in $\ell$ is explicit) which are given by

$\left\{\begin{array}{l}\operatorname{div} \boldsymbol{\sigma}[\ell]+\overline{\mathbf{f}}=\mathbf{0}, \quad \boldsymbol{\sigma}[\ell]=\lambda \operatorname{div} \mathbf{u}[\ell] \mathbf{I}+2 \mu \boldsymbol{\varepsilon}(\mathbf{u}[\ell]) \quad \text { in } \quad \Omega \backslash \Gamma[\ell], \\ \boldsymbol{\sigma}[\ell] \mathbf{n}=\overline{\mathbf{F}} \text { on } \partial_{F} \Omega, \quad \mathbf{u}[\ell] \mathbf{n}=\overline{\boldsymbol{\xi}} \quad \text { on } \quad \partial_{D} \boldsymbol{\Omega}, \\ \boldsymbol{\sigma}[\ell] \mathbf{n}=\sigma[\ell]_{n n} \mathbf{n}, \quad \sigma[\ell]_{n n} \leq 0, \quad \llbracket u[\ell]_{n} \rrbracket \geq 0, \quad \sigma[\ell]_{n n} \llbracket u[\ell]_{n} \rrbracket=0 \quad \text { on } \quad \Gamma[\ell]\end{array}\right.$

with always the mode I assumption and $\Gamma[\ell]=\{\hat{\mathbf{x}}(s) \in$ $\hat{\Gamma}: s \in[0, \ell]\}$. The set of Eq. (21) corresponds to an elastic problem posed on the cracked domain with a (non cohesive) crack of length $\ell$ and with a loading given by the data $(\overline{\mathbf{f}}, \overline{\mathbf{F}}, \bar{\xi})$. The displacement $\mathbf{u}[\ell]$ will be in general singular at the tip $\hat{\mathbf{x}}(\ell)$ of the crack with the usual singularity in $\sqrt{r}$. Specifically, because of the mode I assumption, the mode II stress intensity factor, say $\mathrm{K}_{\mathrm{II}}[\ell]$, vanishes and $\mathbf{u}[\ell](\mathbf{x})$ in the neighborhood of the tip of the crack can read as

$\mathbf{u}[\ell](\mathbf{x})=\frac{\mathrm{K}_{\mathrm{I}}[\ell]}{2 \mu} \sqrt{\frac{r}{2 \pi}} \mathbf{u}^{S}(\theta)+\mathbf{u}[\ell]^{R}(\mathbf{x})$

with

$\mathbf{u}^{S}(\theta)=(3-4 v-\cos \theta)\left(\cos \frac{\theta}{2} \mathbf{t}(\ell)+\sin \frac{\theta}{2} \mathbf{n}(\ell)\right)$

where $r$ denotes the distance of $\mathbf{x}$ to $\hat{\mathbf{x}}(\ell), \theta$ is the angle made by $\mathbf{x}-\hat{\mathbf{x}}(\ell)$ with the tangent $\mathbf{t}(\ell)$ to $\hat{\Gamma}$ at $\hat{\mathbf{x}}(\ell), \mathbf{n}(\ell)=\mathbf{e}_{3} \wedge \mathbf{t}(\ell)$. In (22) $\mathbf{u}[\ell]^{R}$ denotes the regular part of $\mathbf{u}[\ell]$, which is locally in $H^{2}$, and the mode I stress intensity factor $\mathrm{K}_{\mathrm{I}}[\ell]$ depends in general on $\ell$. Because of the non interpenetration condition, $\mathrm{K}_{\mathrm{I}}[\ell]$ is necessarily non negative and we shall assume that it is positive, what means that $\mathbf{u}[\ell]$ is really singular.

H5 The effective stress intensity factor is positive: $\mathrm{K}_{\mathrm{I}}[\ell]>0$.

Of course, this property is a global property which must be checked for each particular problem and each crack length. The consequence is that the crack is necessarily open in a neighborhood $(\ell-h, \ell)$ of the crack tip. Therefore, $\llbracket u[\ell]_{n} \rrbracket(s)>0$ and $\sigma[\ell]_{n n}(s)=0$ for $s \in(\ell-h, \ell)$.

Remark 5 For $\varepsilon$ sufficiently small, the condition $\sigma_{n n}^{2 N_{\epsilon}-1} \leq \sigma_{c}$ is satisfied by its approximation $\sqrt{\epsilon} \boldsymbol{\sigma}[\ell]$ everywhere on $\hat{\Gamma} \backslash \Gamma[\ell]$, except near the tip $\hat{\mathbf{x}}(\ell)$, provided that $\boldsymbol{\sigma}[\ell]$ is not singular at another point of the crack path.

In terms of energy, the real potential energy (difference between the strain energy and the work of the external forces) of the body at time $t=2 N_{\varepsilon}-1$ is well approximated by the potential energy associated with the field $\mathbf{u}[\ell]$, i.e.,

$\mathcal{P}^{2 N_{\epsilon}-1}=\epsilon \mathcal{P}[\ell]+o(\epsilon)$ 
with

$$
\begin{aligned}
\mathcal{P}[\ell]= & \int_{\Omega \backslash \hat{\Gamma}} \frac{1}{2} \mathrm{~A} \boldsymbol{\varepsilon}(\mathbf{u}[\ell]) \cdot \boldsymbol{\varepsilon}(\mathbf{u}[\ell]) d x-\int_{\Omega} \overline{\mathbf{f}} \cdot \mathbf{u}[\ell] d x \\
& -\int_{\partial_{F} \Omega} \overline{\mathbf{F}} \cdot \mathbf{u}[\ell] d s .
\end{aligned}
$$

Defining, as usually, the potential energy release rate $\mathrm{G}[\ell]$ as $-\frac{d \mathcal{P}[\ell]}{d \ell}$, we can use Irwin's formula to link $\mathrm{G}[\ell]$ with $\mathrm{K}_{\mathrm{I}}[\ell]$ :

$\mathrm{G}[\ell]:=-\frac{d \mathcal{P}[\ell]}{d \ell}=\frac{1-v^{2}}{E} \mathrm{~K}_{\mathrm{I}}[\ell]^{2}$.

Note however that this concept of energy release rate is well defined only for the "limit" problem, i.e., when the process zone is neglected.

Let $\vartheta \in \mathbb{R}$ be given and independent of $\varepsilon$. By Hypotheses H2-H3, the position of the non cohesive crack tip at time $t=2 N_{\varepsilon}-1+\vartheta$ differs from $\ell$ only by a term of the order of $\varepsilon$ and the length of the process zone is of the order of $\varepsilon$. Hence the true displacement and stress fields at that time can be well approximated by $\left(\sqrt{\epsilon} \mathbf{u}[\ell]^{\vartheta}, \sqrt{\epsilon} \boldsymbol{\sigma}[\ell]^{\vartheta}\right)$ which are given by a set of equations similar to (21) the loading being now $(\varpi(\vartheta+1) \overline{\mathbf{f}}, \varpi(\vartheta+1) \overline{\mathbf{F}}, \varpi(\vartheta+1) \bar{\xi})$ with $\varpi$ given by $(20)$. By virtue of the linear character of this elastic problem, we have $\mathbf{u}[\ell]^{\vartheta}=\varpi(\vartheta+1) \mathbf{u}[\ell]$. That means that, at a macro scale, neglecting the process zone and the propagation of the crack during a micro number of cycles, the response of the body oscillates because of the periodicity of the loading. Therefore, the stress intensity factor $\mathrm{K}_{\mathrm{I}}[\ell]^{\vartheta}$ oscillates between 0 and $\mathrm{K}_{\mathrm{I}}[\ell]$ : $\mathrm{K}_{\mathrm{I}}[\ell]^{\vartheta}=\varpi(\vartheta+1) \mathrm{K}_{\mathrm{I}}[\ell]$.

\subsection{The small scale problem}

\subsubsection{The rescaling and the stationary regime assumption}

The field $\sqrt{\epsilon} \mathbf{u}[\ell]$ is a good approximation of $\mathbf{u}^{2 N_{\epsilon}-1}$ only far enough from the tip $\hat{\mathbf{x}}(\ell)$. This approximation is sufficient for evaluating the energy of the whole body at this time, but not to determine the evolution of the crack from one cycle to the other. For that it is necessary to take account of the process zone and of the cumulative process of the opening during a cycle. We have to refine the analysis by considering the problem at a small scale, both in space and time. (Figs. 1, 2 and 3)

Let us change the time origin by setting $\vartheta=t-$ $2 N_{\varepsilon}+1$. The rescaled times $\vartheta=2 i, i \in \mathbb{Z}$, correspond to the end of the loading phases of the $i$ th cycle after (or before when $i$ is negative) the cycle $N_{\varepsilon}$. Let us make a zoom of the crack tip $\hat{\mathbf{x}}(\ell)$ by introducing the new cartesian coordinate system $(x, y)$ where $(0,0)$ corresponds to the tip $\hat{\mathbf{x}}(\ell)$ and the axis $x$ corresponds to the direction of the tangent $\mathbf{t}(\ell)$ to $\hat{\Gamma}$ at $\hat{\mathbf{x}}(\ell)$ :

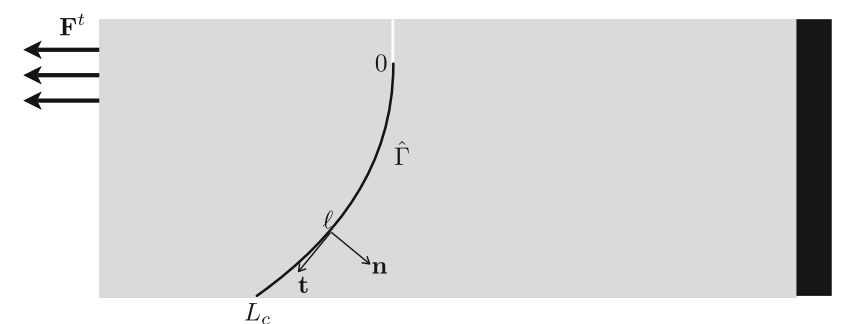

Fig. 1 The body $\Omega$ with the predefined crack path $\hat{\Gamma}$ and its parameterization

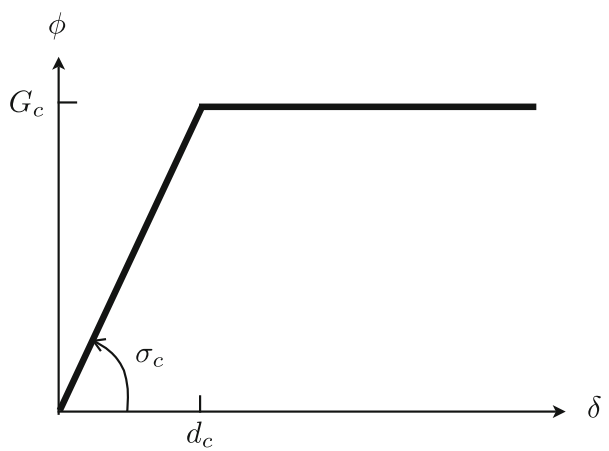

Fig. 2 Dugdale's surface energy density

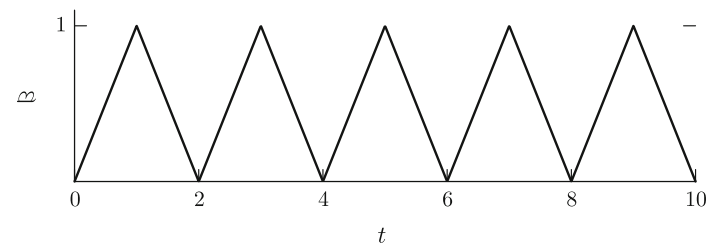

Fig. 3 Simple cyclic loading

$\mathbf{x}-\hat{\mathbf{x}}(\ell)=\epsilon(x \mathbf{t}(\ell)+y \mathbf{n}(\ell)), \quad \mathbf{t}(\ell)=\frac{d \hat{\mathbf{x}}}{d s}(\ell)$,
$\mathbf{n}(\ell)=\mathbf{e}_{3} \wedge \mathbf{t}(\ell)$.

The small scale domain is then the plane $\mathbb{R}^{2}$ and the crack path is the axis $y=0$. For a given $\vartheta$ independent of $\varepsilon$, denoting by $\mathbf{U}^{\vartheta}, \boldsymbol{\Sigma}^{\vartheta}$ and $\Delta^{\vartheta}$ the approximation of the displacement, stress and cumulated opening fields near the crack tip $\hat{\mathbf{x}}(\ell)$, we have

$\left\{\begin{array}{l}\mathbf{u}^{2 N_{\epsilon}-1+\vartheta}(\mathbf{x})=\varpi(\vartheta+1) \sqrt{\epsilon} \mathbf{u}[\ell](\hat{\mathbf{x}}(\ell))+\epsilon \mathbf{U}^{\vartheta}(x, y)+o(\epsilon) \\ \boldsymbol{\sigma}^{2 N_{\epsilon}-1+\vartheta}(\mathbf{x})=\boldsymbol{\Sigma}^{\vartheta}(x, y)+o(1) \\ \delta^{2 N_{\epsilon}-1+\vartheta}(\ell+\epsilon x)=\epsilon \Delta^{\vartheta}(x)+o(\epsilon)\end{array}\right.$.

With these notations, we are in a position to formulate precisely Hypothesis $\mathbf{H 4}$ and the concept of stationary regime. Specifically, we assume that

$$
\begin{gathered}
\forall \vartheta \in[0,2), \forall i \in \mathbb{Z}, \quad \mathbf{U}^{2 i+\vartheta}(x, y)=\mathbf{U}^{\vartheta}(x-i \dot{\ell}, y), \\
\Delta^{2 i+\vartheta}(x)=\Delta^{\vartheta}(x-i \dot{\ell})
\end{gathered}
$$


which means that from a cycle to the other the fields are simply shifted in the direction $x$ through the crack growth rate $\dot{\ell}$. Therefore, the length of the process zone and the growth rate of the non cohesive crack tip are, at first approximation, 2-periodic in $\vartheta$ :

$\mathrm{d}^{2 N_{\epsilon}-1+\vartheta}=\epsilon D^{\vartheta}+o(\epsilon), \quad D^{\vartheta+2 i}=D^{\vartheta}$,

$\forall \vartheta \in \mathbb{R}, \forall i \in \mathbb{Z}$

$\ell^{2 N_{\epsilon}-1+\vartheta}=\ell+\epsilon L^{\vartheta}+o(\epsilon)$,

$L^{\vartheta+2 i}=i \dot{\ell}+L^{\vartheta}, \quad \forall \vartheta \in \mathbb{R}, \forall i \in \mathbb{Z}$

and, by definition, $L^{0}=0$. By virtue of this a priori assumption, it suffices to determine $\dot{\ell}$ and $\mathbf{U}^{\vartheta}, \boldsymbol{\Sigma}^{\vartheta}, \Delta^{\vartheta}, D^{\vartheta}, L^{\vartheta}$ for $\vartheta \in[0,2)$. Let us recall for further reference the full set of relations they must satisfy: the two approximations of the displacement field $\mathbf{u}^{2 N_{\epsilon}-1+\vartheta}$, the behavior of $\varepsilon \mathbf{U}^{\vartheta}$ at infinity is given by the behavior of the singular part of $\sqrt{\epsilon} \mathbf{u}[\ell]^{\vartheta}$ at $\hat{\mathbf{x}}(\ell)$. Specifically, close to $\hat{\mathbf{x}}(\ell)$ we have

$$
\begin{aligned}
\sqrt{\epsilon} \mathbf{u}[\ell]^{\vartheta}(\hat{\mathbf{x}}(\ell)+\epsilon(x \mathbf{t}+y \mathbf{n}))= & \varpi(\vartheta+1) \sqrt{\epsilon} \mathbf{u}[\ell](\hat{\mathbf{x}}(\ell)) \\
& +\varpi(\vartheta+1) \epsilon \frac{\mathrm{K}_{\mathrm{I}}[\ell]}{2 \mu} \sqrt{\frac{r}{2 \pi}} \mathbf{u}^{S}(\theta) \\
& +o(\epsilon)
\end{aligned}
$$

with $x=r \cos \theta$ and $y=r \sin \theta$. Comparing (30) with the first of (25) yields the last of (29).

Notation In the next subsections up to Sect. 3.4, since $\ell$ is fixed and plays the role of a parameter, we remove it from the notations.

$$
\left\{\begin{array}{l}
L^{0}=0, \quad L^{\vartheta} \geq 0, \quad D^{\vartheta}>0 \\
\mathbf{\Sigma}^{\vartheta}=\lambda \operatorname{div} \mathbf{U}^{\vartheta} \mathbf{I}+2 \mu \boldsymbol{\varepsilon}\left(\mathrm{U}^{\vartheta}\right), \quad \operatorname{div} \Sigma^{\vartheta}=\mathbf{0} \quad \text { in } \quad \mathbb{R}^{2} \backslash\left(-\infty, L^{\vartheta}+D^{\vartheta}\right) \times\{0\} \\
\Gamma_{\mathrm{D}}^{\vartheta}:=\left(-\infty, L^{\vartheta}\right) \times\{0\}, \quad \Gamma_{\mathrm{C}}^{\vartheta}:=\left(L^{\vartheta}, L^{\vartheta}+D^{\vartheta}\right) \times\{0\}, \quad \Gamma_{\mathrm{B}}^{\vartheta}:=\left(L^{\vartheta}+D^{\vartheta},+\infty\right) \times\{0\} \\
\Gamma_{\mathrm{Ca}}^{\vartheta}=\Gamma_{\mathrm{C}}^{\vartheta} \cap\left\{\llbracket \dot{U}_{2}^{\vartheta} \rrbracket 0\right\}, \quad \Gamma_{\mathrm{Cn}}^{\vartheta}=\Gamma_{\mathrm{C}}^{\vartheta} \cap\left\{\llbracket \dot{U}_{2}^{\vartheta} \rrbracket=0\right\}, \quad \Gamma_{\mathrm{Cp}}^{\vartheta}=\Gamma_{\mathrm{C}}^{\vartheta} \cap\left\{\llbracket \dot{U}_{2}^{\vartheta} \rrbracket<0\right\} \\
\Sigma_{22}^{\vartheta}=0, \quad \llbracket U_{2}^{\vartheta} \rrbracket \geq 0 \quad \text { on } \quad \Gamma_{\mathrm{D}}^{\vartheta} \\
\Sigma_{22}^{\vartheta}=\sigma_{c}, \llbracket U_{2}^{\vartheta} \rrbracket>\quad \text { on } \Gamma_{\mathrm{Ca}}^{\vartheta}, \quad \Sigma_{22}^{\vartheta} \in\left[0, \sigma_{c}\right], \llbracket U_{2}^{\vartheta} \rrbracket \geq 0 \quad \text { on } \Gamma_{\mathrm{Cn}}^{\vartheta}, \quad \Sigma_{22}^{\vartheta}=0, \llbracket U_{2}^{\vartheta} \rrbracket \geq 0 \quad \text { on } \Gamma_{\mathrm{Cp}}^{\vartheta} \\
\Sigma_{22}^{\vartheta} \leq \sigma_{c}, \quad \llbracket U_{2}^{\vartheta} \rrbracket=0 \quad \text { on } \quad \Gamma_{\mathrm{B}}^{\vartheta} \\
\dot{\Delta}^{\vartheta}=\llbracket \dot{U}_{2}^{\vartheta} \rrbracket^{+} \quad \text { on } \quad y=0 \\
\Delta^{\vartheta}>L_{c} \text { on } \Gamma_{\mathrm{D}}^{\vartheta}, \quad 0<\Delta^{\vartheta}<L_{c} \quad \text { on } \Gamma_{\mathrm{C}}^{\vartheta}, \quad \Delta^{\vartheta}=0 \quad \text { on } \Gamma_{\mathrm{B}}^{\vartheta} \\
\lim _{r \rightarrow \infty}\left(\mathbf{U}^{\vartheta}(r \cos \theta, r \sin \theta)-\varpi(\vartheta+1) \frac{\mathrm{K}_{\mathrm{I}}[\ell]}{2 \mu} \sqrt{\frac{r}{2 \pi}} \mathbf{u}^{S}(\theta)\right)=0
\end{array}\right.
$$

The structure of the solution that we construct in the next subsections is indicated in Fig. 4. Once the solution is found in the interval $[0,2)$, it can be periodically extended to all $\mathbb{R}$ in order that (26)-(28) be automatically satisfied. However, it could happen that the so constructed solution be discontinuous at $\vartheta=2 i$ for $i \in \mathbb{Z}$ and hence we shall also check the continuity of $\mathbf{U}^{\vartheta}, \mathbf{\Sigma}^{\vartheta}, \Delta^{\vartheta}, D^{\vartheta}$ and $L^{\vartheta}$ at $\vartheta=2$. Note that the boundary conditions on the crack line have been simplified by assuming that there are no contact forces between the crack lips owing to the assumption that $\mathrm{K}_{\mathrm{I}}[\ell]>0$. The last equation of (29) giving the behavior of $\mathbf{U}^{\vartheta}$ at infinity can be justified as follows. In order to match

\subsubsection{Determination of $\mathbf{U}^{0}, \mathbf{\Sigma}^{0}$ and $D^{O}$}

At small scale and at $\vartheta=0$, the non cohesive crack is the halfline $x<0$ whereas the process zone is an interval of length $D^{0}$ : $\Gamma_{\mathrm{D}}^{0}=(-\infty, 0) \times\{0\}, \quad \Gamma_{\mathrm{C}}^{0}=\left(0, D^{0}\right) \times\{0\}$, $\Gamma_{\mathrm{B}}^{0}=\left[D^{0},+\infty\right) \times\{0\}$.

We search the solution such that all the process zone is active (and open) at the end of each loading phase, i.e., $\Gamma_{\mathrm{Ca}}^{0}=\Gamma_{\mathrm{C}}^{0}$. The checking of this hypothesis needs to determine the evolution of the displacement field $\mathbf{U}^{9}$ during a cycle. That will be made in the next subsections. Accordingly, $\mathbf{U}^{0}$ and $\boldsymbol{\Sigma}^{0}$ are such that

$\left\{\begin{array}{l}\operatorname{div} \mathbf{\Sigma}^{0}=0, \quad \mathbf{\Sigma}^{0}=\lambda \operatorname{div} \mathbf{U}^{0} \mathbf{I}+2 \mu \boldsymbol{\varepsilon}\left(\mathbf{U}^{0}\right) \text { in } \mathbb{R}^{2} \backslash\left(-\infty, D^{0}\right) \times\{0\} \\ \mathbf{\Sigma}^{0} \mathbf{e}_{2}=0 \quad \text { on } \Gamma_{\mathrm{D}}^{0}, \quad \mathbf{\Sigma}^{0} \mathbf{e}_{2}=\sigma_{c} \mathbf{e}_{2} \quad \text { on } \Gamma_{\mathrm{C}}^{0}, \quad \Sigma_{22}^{0} \leq \sigma_{c} \text { on } \Gamma_{\mathrm{B}}^{0} \\ \llbracket U_{2}^{0} \rrbracket \geq 0 \quad \text { on } \Gamma_{\mathrm{D}}^{0}, \quad \llbracket U_{2}^{0} \rrbracket \geq 0 \quad \text { on } \Gamma_{\mathrm{C}}^{0}, \llbracket U_{2}^{0} \rrbracket=0 \quad \text { on } \Gamma_{\mathrm{B}}^{0} \\ \lim _{r \rightarrow \infty}\left(\mathbf{U}^{0}(r \cos \theta, r \sin \theta)-\frac{\mathrm{K}_{\mathrm{I}}}{2 \mu} \sqrt{\frac{r}{2 \pi}} \mathbf{u}^{S}(\theta)\right)=0 .\end{array}\right.$ 


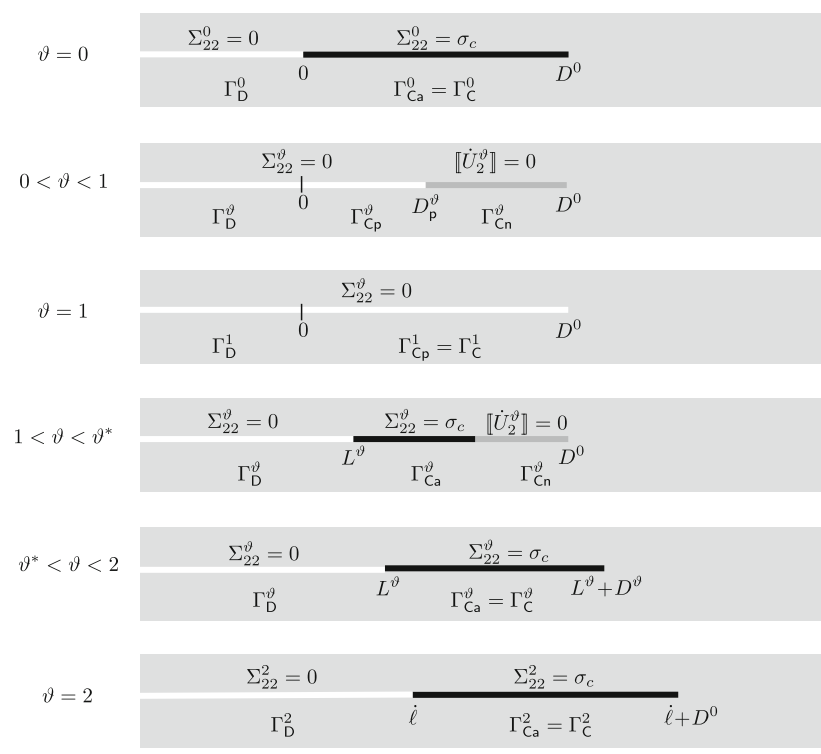

Fig. 4 Evolution of the non cohesive crack and of the cohesive crack during a cycle: in white, the non cohesive crack or the passive part of the cohesive crack; in gray, the neutral part of the cohesive crack; in black, the active part of the cohesive crack. The associated boundary conditions in terms of the normal stress or of the opening rate are indicated. The main stages of the evolution are as follows: at $\vartheta=0$, all the cohesive crack is active and the cohesive stress is equal to $\sigma_{c}$; during the unloading phase, $0<\vartheta<1$, the cohesive crack becomes progressively passive, the cohesive stress is equal to 0 in the passive part while the opening does not evolve in the neutral part; at $\vartheta=1$ which corresponds to the end of the unloading phase, all the cohesive crack is passive; during the first part of the loading phase, $1<\vartheta<\vartheta^{*}$, the tip of the non cohesive crack propagates while the tip of the cohesive crack does not evolve, a part of the cohesive crack remains neutral, the cohesive stress is equal to $\sigma_{c}$ in the active part while the opening does not evolve in the neutral part; at $\vartheta=\vartheta^{*}$, all the cohesive crack is active; during the second part of the loading phase, $\vartheta^{*}<\vartheta<2$, all the cohesive crack is active, both the tip of the cohesive crack and the tip of the non cohesive crack propagate; at $\vartheta=2$, the tip of the non active crack is at $\dot{\ell}$ and the length of the cohesive crack is $D^{0}$ again

The set of Eq. (31) constitutes a generic problem treated in the "Appendix 1" with parameters $\mathrm{L}=0, \mathrm{D}=D^{0}$ and $\mathrm{K}=\mathrm{K}_{\mathrm{I}}$. Thus, the stress intensity factor $\mathrm{K}_{\mathrm{I}}$ plays the role of the intensity of the loading for the small scale problem. Since the normal stress is bounded by $\sigma_{c}$ on $\Gamma_{\mathrm{B}}^{0}$ and because of the non interpenetration condition, there is no singularity at the tip $x=D^{0}$ of the process zone and therefore the length of the process zone is related to $\mathrm{K}_{\mathrm{I}}$ by

$D^{0}=\frac{\pi \mathrm{K}_{\mathrm{I}}^{2}}{8 \sigma_{c}^{2}}=\frac{\pi E \mathrm{G}}{8\left(1-v^{2}\right) \sigma_{c}^{2}}$.

Moreover the jump of the normal displacement on the crack line is given by

$\llbracket U_{2}^{0} \rrbracket(x)=V\left(\frac{x}{D^{0}}\right) \frac{\mathrm{G}}{\sigma_{c}}$ where $V$ is given by (55) and G denotes the rescaled potential energy release rate, see (23). The normal stress on $\Gamma_{\mathrm{B}}^{0}$ is given by

$\Sigma_{22}^{0}(x, 0)=\left(1-\frac{2}{\pi} \arcsin \sqrt{1-\frac{D^{0}}{x}}\right) \sigma_{c}$.

The non interpenetration condition and the yield stress condition are hence satisfied.

\subsubsection{Determination of $\mathbf{U}^{\vartheta}, \mathbf{\Sigma}^{\vartheta}, L^{\vartheta}$ and $D^{\vartheta}$ when $0<\vartheta \leq 1$}

During the unloading phase of the cycle, the stress intensity factor decreases to $0: \mathrm{K}_{\mathrm{I}}^{\vartheta}=(1-\vartheta) \mathrm{K}_{\mathrm{I}}$. We search a solution such that neither the non cohesive zone nor the process zone propagate. However, the process zone will become progressively passive. Specifically, we search a solution such that

$$
\begin{gathered}
L^{\vartheta}=0, \quad D^{\vartheta}=D^{0}, \quad \Gamma_{\mathrm{Cp}}^{\vartheta}=\left(0, D_{\mathrm{p}}^{\vartheta}\right) \times\{0\}, \\
\Gamma_{\mathrm{Cn}}^{\vartheta}=\left(D_{\mathrm{p}}^{\vartheta}, D^{0}\right) \times\{0\}
\end{gathered}
$$

with $\vartheta \mapsto D_{\mathrm{p}}^{\vartheta}$ growing from 0 to $D^{0}$. Accordingly, $\mathbf{U}^{\vartheta}$ and $\boldsymbol{\Sigma}^{\vartheta}$ have to satisfy the following conditions on the crack line:

$$
\begin{aligned}
& \text { on } \Gamma_{\mathrm{D}}^{\vartheta}\left\{\begin{array} { l } 
{ \Sigma _ { 2 2 } ^ { \vartheta } = 0 } \\
{ \llbracket U _ { 2 } ^ { \vartheta } \rrbracket \geq 0 }
\end{array} \quad \text { on } \Gamma _ { \mathrm { Cp } } ^ { \vartheta } \left\{\begin{array}{c}
\Sigma_{22}^{\vartheta}=0 \\
0 \leq \llbracket U_{2}^{\vartheta} \rrbracket \leq \llbracket U_{2}^{0} \rrbracket
\end{array}\right.\right. \\
& \text { on } \Gamma_{\mathrm{Cn}}^{\vartheta}\left\{\begin{array} { l } 
{ 0 \leq \Sigma _ { 2 2 } ^ { \vartheta } \leq \sigma _ { c } } \\
{ \llbracket U _ { 2 } ^ { \vartheta } \rrbracket = \llbracket U _ { 2 } ^ { 0 } \rrbracket }
\end{array} , \quad \text { on } \Gamma _ { \mathrm { B } } ^ { \vartheta } \left\{\begin{array}{c}
\Sigma_{22}^{\vartheta} \leq \sigma_{c} \\
\llbracket U_{2}^{\vartheta} \rrbracket=0
\end{array}\right.\right.
\end{aligned}
$$

Setting $\mathbf{U}^{\vartheta}=\mathbf{U}^{0}-\overline{\mathbf{U}}^{\vartheta}$ and $\boldsymbol{\Sigma}^{\vartheta}=\boldsymbol{\Sigma}^{0}-\overline{\mathbf{\Sigma}}^{\vartheta}$, the pair $\left(\overline{\mathbf{U}}^{\vartheta}, \overline{\mathbf{\Sigma}}^{\vartheta}\right)$ is solution of the generic problem of "Appendix 1" with parameters $\mathrm{K}=\vartheta \mathrm{K}_{\mathrm{I}}, \mathrm{L}=0$ and $\mathrm{D}=D_{\mathrm{p}}^{\vartheta}$. Moreover $\overline{\boldsymbol{\Sigma}}^{\vartheta}$ and $\overline{\mathbf{U}}^{\vartheta}$ must be such that $0 \leq \bar{\Sigma}_{22}^{\vartheta} \leq \sigma_{c}$ on $\Gamma_{\mathrm{Cn}}^{\vartheta}$ and $\llbracket \bar{U}_{2}^{\vartheta} \rrbracket \geq 0$ on $\Gamma_{\mathrm{Cp}}^{\vartheta}$. (Note that the condition $\llbracket \bar{U}_{2}^{\vartheta} \rrbracket \geq 0$ is not due to the non interpenetration condition but to the decrease of the opening during the unloading phase.) Therefore, there is no singularity at the tip of the neutral zone, its position is given by $D_{\mathrm{p}}^{\vartheta}=$ $\vartheta^{2} D^{0}$ and grows from 0 to $D^{0}$ during the unloading phase. The jump of the normal displacement on the crack line is given by

$\llbracket U_{2}^{\vartheta} \rrbracket(x)=\left(V\left(\frac{x}{D^{0}}\right)-\vartheta^{2} V\left(\frac{x}{\vartheta^{2} D^{0}}\right)\right) \frac{\mathrm{G}}{\sigma_{c}}$

while the normal stress on the neutral zone and the bonded zone is given by

$\Sigma_{22}^{\vartheta}(x, 0)= \begin{cases}\sigma_{c} \frac{2}{\pi} \arcsin \sqrt{1-\frac{v^{2} D^{0}}{x}} & \text { if } \vartheta^{2} D^{0} \leq x \leq D^{0} \\ \sigma_{c} \frac{2}{\pi}\left(\arcsin \sqrt{1-\frac{v^{2} D^{0}}{x}}-\arcsin \sqrt{1-\frac{D^{0}}{x}}\right) & \text { if } x \geq D^{0}\end{cases}$

Let us now verify all the required conditions for $\mathbf{U}^{\vartheta}$ and $\boldsymbol{\Sigma}^{\vartheta}$. First, $\lim _{\vartheta \downarrow 0}\left(\mathbf{U}^{\vartheta}, \mathbf{\Sigma}^{\vartheta}\right)=\left(\mathbf{U}^{0}, \mathbf{\Sigma}^{0}\right)$. Second, since $V$ is strictly decreasing on $[0,1], \llbracket \dot{U}_{2}^{\vartheta} \rrbracket(x)<0$ when $0<x<\vartheta^{2} D^{0}$, in 
conformity with the definition of $\Gamma_{\mathrm{Cp}}^{\vartheta}$. Third, since $V$ is concave on $(-\infty, 0)$ with $V(0)=1$, we have $V(\theta \zeta)-\theta$ $V(\zeta) \geq(1-\theta) V(0) \geq 0$ for $\zeta \leq 0$ and $0<\theta \leq 1$; hence (34) yields $\llbracket U_{2}^{\vartheta} \rrbracket(x) \geq 0$ when $x \leq 0$. Since $V$ is decreasing and positive on $[0,1]$, we have $V(\theta \zeta) \geq V(\zeta) \geq \theta V(\zeta)$ for $0 \leq \zeta \leq 1$ and $0<\theta \leq 1$; hence (34) yields $\llbracket U_{2}^{\vartheta} \rrbracket(x) \geq 0$ when $0 \leq x \leq \vartheta^{2} D^{0}$. Therefore, the non interpenetration condition is satisfied everywhere. Finally, it is immediate from (35) that $0 \leq \Sigma_{22}^{\vartheta}(x, 0) \leq \sigma_{c}$ when $x \geq \vartheta^{2} D^{0}$. All the conditions are satisfied by $\mathbf{U}^{\vartheta}$ and $\boldsymbol{\Sigma}^{\vartheta}$.

When $\vartheta \uparrow 1$, all the process zone becomes passive, the length of the neutral zone $\left(1-\vartheta^{2}\right) D^{0}$ tends to 0 . We can see in (35) (or in Fig. 5) that $\lim _{\vartheta \uparrow 1} \Sigma_{22}^{\vartheta}(x, 0)=0$ for all $x$. Since $V(\zeta)=2 \sqrt{|\zeta|}+o(1)$ when $\zeta \rightarrow-\infty$, (34) gives that $\lim _{\vartheta \uparrow 1} \llbracket U_{2}^{\vartheta} \rrbracket(x)=0$ for all $x$ (see also Fig. 5). Therefore, defining $\left(\mathbf{U}^{1}, \boldsymbol{\Sigma}^{1}\right)$ as the limit of $\left(\mathbf{U}^{\vartheta}, \boldsymbol{\Sigma}^{\vartheta}\right)$ when $\vartheta \uparrow 1$, we have $\mathbf{U}^{1}=\mathbf{0}$ and $\boldsymbol{\Sigma}^{1}=\mathbf{0}$.

Remark 6 Since $\llbracket U_{2}^{\vartheta} \rrbracket(x)$ decreases as $\vartheta$ increases from 0 to 1 , the cumulated opening does not evolve during the unloading phase, $\Delta^{\vartheta}=\Delta^{0}$. Moreover, at the end of the unloading phase, all the crack is closed and free of any contact or cohesive force. Note that $\left(\mathrm{U}^{1}, \Sigma^{1}\right)$ are defined by passage to the limit when $\vartheta \uparrow 1$. We shall see that $\llbracket \dot{U}_{2}^{\vartheta} \rrbracket$ is not defined at $\vartheta=1$ because of the change of the sense of loading, only the left and the right derivatives are defined. It is the same at $\vartheta=2$. Accordingly, one should modify the definition of active, passive or neutral zones at these times by considering the left derivative of $\llbracket U_{2}^{\vartheta} \rrbracket$ (rather than the right derivative for causality reasons). With this new definition, one can prove that $\mathbf{U}^{1}=\mathbf{0}$ and $\boldsymbol{\Sigma}^{1}=\mathbf{0}$ and hence their left-continuity at $\vartheta=1$.

\subsubsection{Determination of $\mathbf{U}^{\vartheta}, \mathbf{\Sigma}^{\vartheta}$ and $D^{\vartheta}$ when $1<\vartheta<2$}

During the loading phase of the cycle, the stress intensity factor increases: $\mathrm{K}_{\mathrm{I}}^{\vartheta}=(\vartheta-1) \mathrm{K}_{\mathrm{I}}$. At $\vartheta=1$ the crack is closed and the process zone is passive. The process zone becomes progressively active and the non cohesive crack tip propagates. Beyond a certain time $\vartheta^{*}$, all the process zone is active and the tip of the process zone propagates. Thus, assuming that $L^{\vartheta}$ is known and continuously increasing with $\vartheta$, we search a solution such that the loading phase itself is divided into two parts as follows:

1. First part of the loading phase: $D^{\vartheta}=D^{0}-L^{\vartheta}, \Gamma_{\mathrm{Ca}}^{\vartheta}=$ $\left(L^{\vartheta}, L^{\vartheta}+D_{\mathrm{a}}^{\vartheta}\right) \times\{0\}, \Gamma_{\mathrm{Cn}}^{\vartheta}=\left(L^{\vartheta}+D_{\mathrm{a}}^{\vartheta}, D^{0}\right) \times\{0\} ;$

2. Second part of the loading phase: $\Gamma_{\mathrm{C}}^{\vartheta}=\Gamma_{\mathrm{Ca}}^{\vartheta}=$ $\left(L^{\vartheta}, L^{\vartheta}+D^{\vartheta}\right) \times\{0\}$.

In both parts, $\mathbf{U}^{\vartheta}$ and $\mathbf{\Sigma}^{\vartheta}$ have to satisfy the following conditions on the crack line:

$\Sigma_{22}^{\vartheta}(x, 0)=0$ if $x<L^{\vartheta}$,

$\Sigma_{22}^{\vartheta}(x, 0)=\sigma_{c}$ if $L^{\vartheta}<x<L^{\vartheta}+D_{\mathrm{a}}^{\vartheta}$,

$\llbracket U_{2}^{\vartheta} \rrbracket(x)=0$ if $x \geq L^{\vartheta}+D_{\mathrm{a}}^{\vartheta}$

with the convention that $D_{\mathrm{a}}^{\vartheta}=D^{\vartheta}$ during the second part. Therefore, the pair $\left(\mathbf{U}^{\vartheta}, \boldsymbol{\Sigma}^{\vartheta}\right)$ is solution of the generic problem of "Appendix 1" with parameters $\mathrm{K}=(\vartheta-1) \mathrm{K}_{\mathrm{I}}, \mathrm{L}=L^{\vartheta}$ and $\mathrm{D}=D_{\mathrm{a}}^{\vartheta}$. Moreover $\Sigma^{\vartheta}$ must be such that $0 \leq \Sigma_{22}^{\vartheta} \leq \sigma_{c}$ on $\Gamma_{\mathrm{Cn}}^{\vartheta}$ and $\Sigma_{22}^{\vartheta} \leq \sigma_{c}$ on $\Gamma_{\mathrm{B}}^{\vartheta}$, whereas $\mathbf{U}^{\vartheta}$ must satisfy the non interpenetration condition. Hence, there is no singularity at $x=L^{\vartheta}+D_{\mathrm{a}}^{\vartheta}, D_{\mathrm{a}}^{\vartheta}$ is given by $D_{\mathrm{a}}^{\vartheta}=(\vartheta-1)^{2} D^{0}$ and grows continuously from 0 to $D^{0}$ during the loading phase. The jump of the normal displacement on the crack line is given by

$\llbracket U_{2}^{\vartheta} \rrbracket(x)=V\left(\frac{x-L^{\vartheta}}{(\vartheta-1)^{2} D^{0}}\right) \frac{(\vartheta-1)^{2} \mathrm{G}}{\sigma_{c}}$

while the normal stress on the neutral zone and the bonded zone is given by

$$
\begin{aligned}
\Sigma_{22}^{\vartheta}(x, 0)= & \sigma_{c}\left(1-\frac{2}{\pi} \arcsin \sqrt{1-\frac{(\vartheta-1)^{2} D^{0}}{x-L^{\vartheta}}}\right) \\
& \text { if } \quad x \geq L^{\vartheta}+(\vartheta-1)^{2} D^{0} .
\end{aligned}
$$

Since $V$ is strictly decreasing on $[0,1]$ and provided that $\vartheta \mapsto L^{\vartheta}$ is continuously increasing, $\llbracket \dot{U}_{2}^{\vartheta} \rrbracket(x)$ is defined and positive for $x \in\left(L^{\vartheta}, L^{\vartheta}+(\vartheta-1)^{2} D^{0}\right)$, in conformity with the definition of $\Gamma_{\mathrm{Ca}}^{\vartheta}$. Furthermore, the non interpenetration condition and the yield stress condition are ensured. The time $\vartheta^{*}$ when the first part of the loading phase finishes is such that $L^{9^{*}}+\left(\vartheta^{*}-1\right)^{2} D^{0}=D^{0}$. Provided that $L^{\vartheta}$ grows continuously when $\vartheta$ grows from 1 to 2 , since $L^{1}=0$, there exists a
Fig. 5 Evolution of the opening and of the normal stress during the unloading phase of a cycle. Note the growth of the passive zone
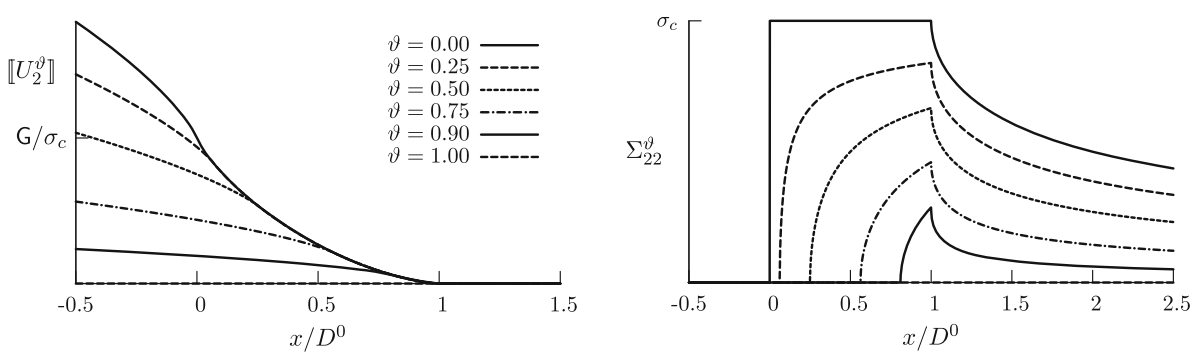
unique $\vartheta^{*}$ satisfying this condition. Since $V(\zeta)=2 \sqrt{|\zeta|}+$ $o(1)$ when $\zeta \rightarrow-\infty$, one gets $\lim _{\vartheta \downarrow}\left(\mathrm{U}^{\vartheta}, \mathbf{\Sigma}^{\vartheta}\right)=(\mathbf{0}, \mathbf{0})$ and hence the continuity at $\vartheta=1$. Provided that $\lim _{\vartheta \uparrow 2} L^{\vartheta}=\dot{\ell}$, it is easy to check that $\lim _{\vartheta \uparrow 2}\left(\mathbf{U}^{\vartheta}, \boldsymbol{\Sigma}^{\vartheta}\right)=\left(\mathbf{U}^{2}, \boldsymbol{\Sigma}^{2}\right)$ with $\left(\mathbf{U}^{2}, \boldsymbol{\Sigma}^{2}\right)$ related to $\left(\mathbf{U}^{0}, \boldsymbol{\Sigma}^{0}\right)$ by the stationary conditions (26).

Remark 7 At this stage, provided that $\vartheta \mapsto L^{\vartheta}$ is continuously increasing on [1,2] from 0 to $\dot{\ell}$, we have found $\mathbf{U}^{\vartheta}$ and $\boldsymbol{\Sigma}^{\vartheta}$ in terms of $L^{\vartheta}$ which satisfy all the requirements for $\vartheta \in[0,2]$. It remains to find $\dot{\ell}, \Delta^{0}$ and $L^{\vartheta}, \Delta^{\vartheta}$ for $\vartheta \in(1,2)$, then, to verify that $\vartheta \mapsto L^{\vartheta}$ and $\vartheta \mapsto \Delta^{\vartheta}$ are continuous at $\vartheta=1$ and $\vartheta=2$.

\subsubsection{Determination of $\Delta^{0}$ and $\dot{\ell}$}

By definition and Hypothesis H1, $\delta^{2 N_{\epsilon}-1}(s)>d_{c}$ for $s<\ell$ and $\delta^{2 N_{\epsilon}-1}(s)<d_{c}$ for $s>\ell$. Hence, if we assume that $\delta^{2 N_{\epsilon}-1}$ is continuous at $s=\ell$, we have $\delta^{2 N_{\epsilon}-1}(\ell)=d_{c}$. Using (24) and (25), we get

$\Delta^{0}(0)=L_{c}$.

By definition (7) of the cumulated opening, since the opening does not increase during the unloading phases and does not decrease during the loading phases, since the opening vanishes at the end of an unloading phase and by virtue of the stationary conditions (26), we have

$$
\begin{aligned}
\Delta^{0}(x) & =\Delta^{-2}(x)+\int_{-2}^{0}\left(\llbracket \dot{U}_{2}^{\vartheta} \rrbracket(x)\right)^{+} d \vartheta \\
& =\Delta^{-2}(x)+\llbracket U_{2}^{0} \rrbracket(x)-\llbracket U_{2}^{-1} \rrbracket(x) \\
& =\Delta^{0}(x+\dot{\ell})+\llbracket U_{2}^{0} \rrbracket(x) .
\end{aligned}
$$

Then, by induction, since $\Delta^{0}(x)=0$ for $x \geq D^{0}$, we get $\Delta^{0}(x)=\sum_{i=0}^{+\infty} \llbracket U_{2}^{0} \rrbracket(x+i \dot{\ell})$. Note that this series contains a priori an infinite number of terms because the micro-number of cycles needed to compute $\Delta^{0}(x)$ depends on $x$ and $\dot{\ell}$, and can tend to infinity when $\dot{\ell}$ goes to 0 . Using (33) we finally obtain

$\Delta^{0}(x)=\sum_{i=0}^{+\infty} V\left(\frac{x+i \dot{\ell}}{D^{0}}\right) \frac{\mathrm{G}}{\sigma_{c}}$

with $D^{0}$ given by (32). Inserting this expression for $\Delta^{0}(0)$ into (37) gives the desired equation for $\dot{\ell}$ :

$\sum_{i=0}^{+\infty} V\left(\frac{i \dot{\ell}}{D^{0}}\right) \mathrm{G}=\sigma_{c} L_{c}$.

This equation will be rewritten and interpreted in the next section by reintroducing the true physical quantities instead of the rescaled ones. Let us analyze here the conditions for the existence and the uniqueness of a solution $\dot{\ell}$ in terms of G. According to whether $\mathrm{G}$ is greater than, equal to or less than $\sigma_{c} L_{c}$ there exists no solution, an infinite number of solutions or a unique solution for $\dot{\ell}$ as it is proved below.

1. If $\mathrm{G}>\sigma_{c} L_{c}$, then there exists no solution for $\dot{\ell}$. Indeed, since $V \geq 0$ and $V(0)=1, \sum_{i=0}^{+\infty} V\left(i \dot{\ell} / D^{0}\right)$ $\mathrm{G} \geq \mathrm{G}>\sigma_{c} L_{c}$ for all $\dot{\ell}$.

2. If $\mathrm{G}=\sigma_{c} L_{c}$, then all the solutions are the $\dot{\ell}$ 's such that $\dot{\ell} \geq \dot{\ell}_{m}:=\frac{\pi E L_{c}}{8\left(1-v^{2}\right) \sigma_{c}}$.

Indeed, in such a case, (39) becomes $\sum_{i=0}^{+\infty} V\left(i \dot{i} / \dot{\ell}_{m}\right)=1$. Since $V(0)=1$, it reads also as $\sum_{i=1}^{+\infty} V\left(i \dot{\ell} / \dot{\ell}_{m}\right)=0$. Since $V \geq 0$ everywhere and $V=0$ only on $[1,+\infty)$, we must have $V\left(\dot{\ell} / \dot{\ell}_{m}\right)=0$ and hence $\dot{\ell} \geq \dot{\ell}_{m}$. Conversely, if $\dot{\ell} \geq \dot{\ell}_{m}$, then $V\left(i \dot{\ell} / \dot{\ell}_{m}\right)=0$ for every $i \geq 1$ and hence $\dot{\ell}$ is solution.

3. If $0<\mathrm{G}<\sigma_{c} L_{c}$, then there exists a unique solution $\dot{\ell}=f(\mathrm{G})>0$.

Indeed, let us consider the function $\lambda \mapsto F(\lambda):=$ $\sum_{i=0}^{+\infty} V\left(i \lambda / D^{0}\right) \mathrm{G}$ defined for $\lambda \geq 0$. Since $V(0)=1$, then $F(0)=+\infty$. Since $V(\zeta)=0$ for $\zeta \geq 1$, then $F(\lambda)=\mathrm{G}<\sigma_{c} L_{c}$ for every $\lambda \geq D^{0}$. When $0<\lambda<D^{0}$, we have $F(\lambda)=\left(1+V\left(\lambda / D^{0}\right)\right) \mathrm{G}+\sum_{i=2}^{+\infty} V\left(i \lambda / D^{0}\right) \mathrm{G}$. Since $V$ is decreasing on $[0,1]$ and vanishes on $[1,+\infty)$, then $F(\lambda)$ decreases from infinity to $\mathrm{G}$ as $\lambda$ goes from 0 to $D^{0}$. Therefore, there exists a unique $\dot{\ell}$ such that $F(\dot{\ell})=\sigma_{c} L_{c}$. Moreover, $\dot{\ell} \in\left(0, D^{0}\right)$.

The precise dependence of $\dot{\ell}$ on $\mathrm{G}$ will be studied in the next section. Let us make the last Hypothesis, which contains Hypothesis $\mathbf{H 5}$,

H6: $0<\mathrm{G}<\sigma_{c} L_{c}$.

In such a case, there exists a unique $\dot{\ell}$ and $\Delta^{0}$ is given by (38). Since $V$ is continuously differentiable, non increasing and non negative, so is $\Delta^{0}$. Moreover, since $V$ is decreasing on $(-\infty, 1]$ and vanishes on $[1,+\infty), \Delta^{0}$ is decreasing on $\left(-\infty, D^{0}\right]$ and vanishes on $\left[D^{0},+\infty\right)$. Accordingly, we have $\Delta^{0}>L_{c}$ on $\Gamma_{\mathrm{D}}^{0}, 0<\Delta^{0}<L_{c}$ on $\Gamma_{\mathrm{C}}^{0}$ and $\Delta^{0}=0$ on $\Gamma_{\mathrm{B}}^{0}$, as it is required.

\subsubsection{Determination of $\Delta^{\vartheta}$ and $L^{\vartheta}$ when $1<\vartheta<2$}

As before, if we assume that $x \mapsto \Delta^{\vartheta}(x)$ is continuous, then $L^{\vartheta}$ is such that

$\Delta^{\vartheta}\left(L^{\vartheta}\right)=L_{c}$.

By definition (7) of the cumulated opening, since the opening does not decrease during the loading phase and since the opening vanishes at $\vartheta=1$, we have

$\Delta^{\vartheta}(x)=\Delta^{1}(x)+\int_{1}^{\vartheta}\left(\llbracket \dot{U}_{2}^{t} \rrbracket(x)\right)^{+} d t=\Delta^{0}(x)+\llbracket U_{2}^{\vartheta} \rrbracket(x)$. 
Inserting this expression for $\Delta^{\vartheta}\left(L^{\vartheta}\right)$ into (40) and using (36) lead to the following equation for $L^{\vartheta}$ :

$\sigma_{c} \Delta^{0}\left(L^{\vartheta}\right)+(\vartheta-1)^{2} \mathrm{G}-\sigma_{c} L_{c}=0$.

Let us set $F(x, \vartheta):=\sigma_{c} \Delta^{0}(x)+(\vartheta-1)^{2} \mathrm{G}-\sigma_{c} L_{c}$ and remark that $F(x, \vartheta)$ decreases from infinity to $(\vartheta-1)^{2} \mathrm{G}-$ $\sigma_{c} L_{c}$ when $x$ goes from $-\infty$ to $D^{0}$, then remains constant for $x \geq D^{0}$. Therefore, since by Hypothesis $\mathbf{H 6}$ $(\vartheta-1)^{2} \mathrm{G}<\sigma_{c} L_{c}$, there exists a unique $L^{\vartheta}$ such that $F\left(L^{\vartheta}, \vartheta\right)=0$. Since $\Delta^{0}(0)=L_{c}$, then $F(0, \vartheta)>0$ and hence $L^{\vartheta} \in\left(0, D^{0}\right)$. Since $F$ is an increasing function of $\vartheta$ at given $x$ and since $\Delta^{0}$ is decreasing on $\left(0, D^{0}\right), \Delta^{0}\left(L^{\vartheta}\right)$ decreases and hence $L^{\vartheta}$ increases when $\vartheta$ is increasing. Moreover, since $V$ is continuously differentiable on $\left[0, D^{0}\right], \vartheta \mapsto L^{\vartheta}$ is continuous on $[1,2]$ and continuously differentiable on $(1,2)$. Therefore, $L^{9}$ grows continuously from 0 to $\dot{\ell}$ during the loading phases. From (41) and by virtue of the continuity of $\vartheta \mapsto \mathbf{U}^{\vartheta}$ at $\vartheta=2$, we get $\lim _{\vartheta \uparrow 2} \Delta^{\vartheta}(x)=\Delta^{0}(x)+\llbracket U_{2}^{2} \rrbracket(x)=\Delta^{2}(x)$ and hence the continuity of $\vartheta \mapsto \Delta^{\vartheta}$ at $\vartheta=2$. Since $\lim _{\vartheta \downarrow 0} \llbracket U_{2}^{\vartheta} \rrbracket=0$, we have also the continuity of $\vartheta \mapsto \Delta^{\vartheta}$ at $\vartheta=1$.

Note that the right derivative of $\vartheta \mapsto \llbracket U_{2}^{\vartheta} \rrbracket(x)$ at $\vartheta=1$ is positive for $x<0$, while its left derivative is negative for $x<D^{0}$. Hence $\vartheta \mapsto \llbracket U_{2}^{\vartheta} \rrbracket(x)$ is not differentiable at $\vartheta=1$. In a similar way, $\vartheta \mapsto \llbracket U_{2}^{\vartheta} \rrbracket(x)$ is not differentiable at $\vartheta=0$ or 2. Its right derivative at $\vartheta=0$ is negative for $x<0$. Its left derivative at $\vartheta=2$ is positive for $x<D^{0}+\dot{\ell}$ and hence its left derivative at $\vartheta=0$ is positive for $x<D^{0}$. Partitioning the process zone by the sign of the left derivative of the opening (see Remark 4), we obtain that all the process zone is active at $\vartheta=0$ as it was assumed.

It remains to verify that the dependence of $\Delta^{\vartheta}$ on $x$ is consistent with the definitions of the three interval $\Gamma_{\mathrm{B}}^{\vartheta}, \Gamma_{\mathrm{C}}^{\vartheta}$ and $\Gamma_{\mathrm{D}}^{\vartheta}$. Using (41) with (36) and (38), it appears that $\Delta^{\vartheta}$ is decreasing on $\left(-\infty, L^{\vartheta}+D^{\vartheta}\right]$ because $\llbracket U_{2}^{\vartheta} \rrbracket$ is decreasing on $\left(-\infty, L^{\vartheta}+D_{\mathrm{a}}^{\vartheta}\right]$ and not increasing otherwise, because $\Delta^{0}$ is decreasing on $\left(-\infty, D^{0}\right]$ and not increasing otherwise, and because $L^{\vartheta}+D^{\vartheta}=\max \left\{D^{0}, L^{\vartheta}+D_{\mathrm{a}}^{\vartheta}\right\}$. Since, by

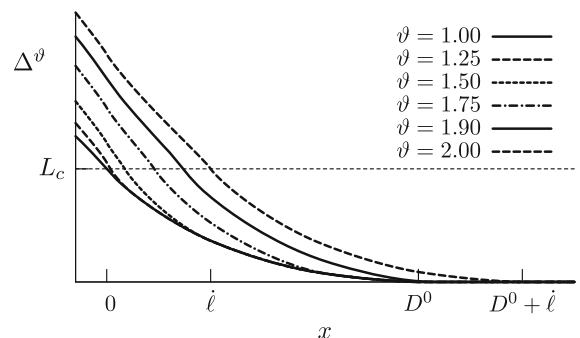

Fig. 6 Evolution of the cumulated opening and of the different zones (non cohesive zone, active zone and process zone) during the loading phase of a cycle when $G / G_{c} \approx 0.636$ (then $\dot{\ell}=D^{0} / 3$ ). Note the two construction, $\Delta^{\vartheta}\left(L^{\vartheta}\right)=L_{c}$ and $\Delta^{\vartheta}\left(L^{\vartheta}+D^{\vartheta}\right)=0$, we have $\Delta^{\vartheta}>L_{c}$ on $\Gamma_{\mathrm{D}}^{\vartheta}, 0<\Delta^{\vartheta}<L_{c}$ on $\Gamma_{\mathrm{C}}^{\vartheta}$ and $\Delta^{\vartheta}=0$ on $\Gamma_{\mathrm{B}}^{\vartheta}$ as required.

Remark 8 Provided that Hypothesis $\mathbf{H 6}$ holds, we have constructed a (smooth) solution for the small scale problem which satisfies the set of conditions (29), see Figs. 5, 6 and 7 where are plotted the evolution of $\llbracket U_{2}^{\vartheta} \rrbracket, \Sigma_{22}^{\vartheta}, \Delta^{\vartheta}, L^{\vartheta}$ and $L^{\vartheta}+D^{\vartheta}$ with $\vartheta$ when $G / G_{c}=\mathrm{G} / \sigma_{c} L_{c} \approx 0.636$. Of course, since no uniqueness result is available, another solution could exist (even in the restricted framework where it was searched). However, the present solution does depend on the large scale problem only through the stress intensity factor and hence can be considered as a universal solution for a crack in mode I in an homogeneous isotropic linear elastic material under a simple cyclic loading.

\subsection{A posteriori verification of the a priori Hypotheses}

To finish this construction of the solution for the evolution problem it remains to check that the a priori assumptions are really satisfied. We reintroduce $\ell$ into the notations. The verification consists in the following procedure.

First, make the following computations:

(I) Compute $\mathbf{u}[\ell], \mathrm{K}_{\mathrm{I}}[\ell], \mathrm{K}_{\mathrm{II}}[\ell], \mathrm{G}[\ell]$ and $\mathbf{u}[\ell]^{R}$ for every $\ell \in\left[0, L_{c}\right)$ by solving $(21)$;

(II) Compute $\dot{\ell}=f(\mathrm{G})$ for every $\mathrm{G} \in\left(0, \sigma_{c} L_{c}\right)$ by solving (39).

Then proceed to the two following verifications:

(i) For every $\ell \in\left[0, L_{c}\right)$, verify that $\mathrm{K}_{\mathrm{II}}[\ell]=0$, that $0<\mathrm{G}[\ell]<\sigma_{c} L_{c}$ and that there exists no other singular point than $\hat{\mathbf{x}}(\ell)$ on $\hat{\Gamma}$;

(ii) Verify that the ordinary differential equation $\frac{d \ell}{d T}=$ $f(\mathrm{G}[\ell])$ with the initial condition $\ell(0)=0$ has a unique solution $T \mapsto \ell(T)$.

Both verifications have a global character and depend on the geometry and on the loading. They can be non satisfied for multiple reasons, for instance:

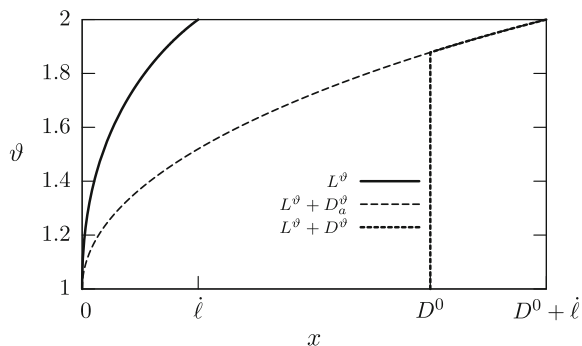

parts of the evolution: the first $\left(\vartheta \leq \vartheta^{*} \approx 1.878\right)$ when the tip of the process zone does not move and the second $\left(\vartheta>\vartheta^{*}\right)$ when both tips propagate 
Fig. 7 Evolution of the opening and of the normal stress during the loading phase of a cycle when $G / G_{c}=0.636$ (then $\left.\dot{\ell}=D^{0} / 3\right)$. Note the growth of the active zone
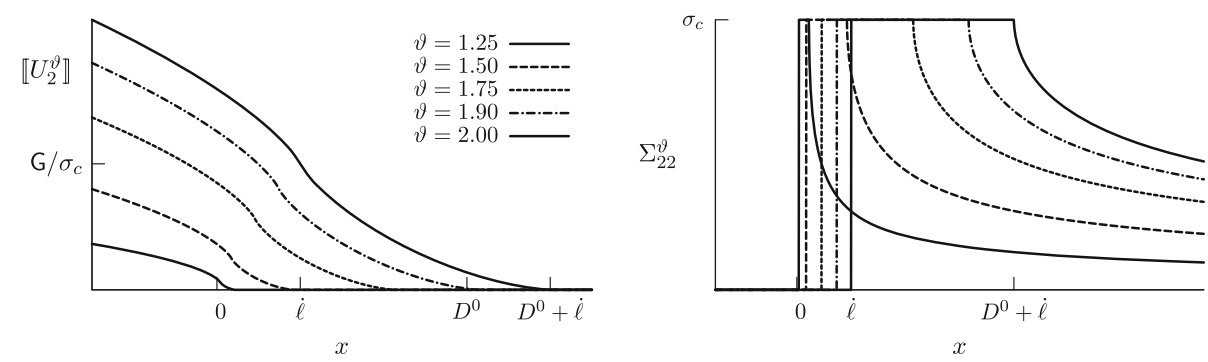

(a) It can happen that the process of fatigue never starts because there is not or not sufficient stress concentration in the uncracked body. In such a case $\mathrm{G}[0]=0$ and $\ell(T)=0$ for all $T \geq 0$ is solution of the differential equation. That means that the nucleation of a crack under cyclic loading requires a specific study (which is outside the scope of our paper);

(b) It can happen that the propagation of the crack becomes "unstable" in the sense that the energy release rate becomes larger than $G_{c}$. In such a case, the propagation of the crack is no more governed by the fatigue law (but it can still be governed by the evolution problem of Sect. 2.2);

(c) It can happen that several crack tips propagate simultaneously.

If they are satisfied then it is possible to construct an approximate solution for the evolution problem as follows $\left(\varepsilon=d_{c} / L_{c}\right.$ is fixed):

1. Let $t>0$. Define $N$ as the integer part of $(t+1) / 2$, $\vartheta=t+1-2 N$ and $T=\varepsilon N$;

2. Set $\ell(T)$ as the solution of the differential equation $\frac{d \ell}{d T}=f(\mathrm{G}[\ell])$ at $T$

3. Compute $\mathbf{U}^{\vartheta}, \Delta^{\vartheta}, D^{\vartheta}$ and $L^{\vartheta}$ for every $\vartheta \in[0,2)$ at the given $T$ (they depend on $\ell(T)$ );

4. Define $\mathbf{u}^{t}, \ell^{t}$ and $\mathrm{d}^{t}$ by

$$
\begin{gathered}
\mathbf{u}^{t}(\mathbf{x})=\varpi(\vartheta+1) \sqrt{\epsilon} \mathbf{u}[\ell(T)]^{R}(\mathbf{x})+\epsilon \mathbf{U}^{\vartheta}(x, y), \\
\mathrm{d}^{t}=\epsilon D^{\vartheta}, \quad \ell^{t}=\ell(T)+\epsilon L^{\vartheta}
\end{gathered}
$$

where

$$
x \mathbf{t}+y \mathbf{n}=\frac{1}{\epsilon}(\mathbf{x}-\hat{\mathbf{x}}(\ell(T))), \quad \mathbf{t}=\frac{d \hat{\mathbf{x}}}{d s}(\ell(T)), \quad \mathbf{n}=\mathbf{e}_{3} \wedge \mathbf{t} .
$$

Then all the hypotheses H1-H6 are satisfied. (Of course, this is not an exact solution because its construction comes from a separation of scales.)

\section{Properties of the fatigue law and its dependence on the cohesive model and the loading}

4.1 The fatigue law corresponding to Dugdale's model, cumulated opening and simple cycle in mode I

Let us reintroduce the real physical quantities instead of the rescaled ones: $\ell$ denoting the position of the tip of the non cohesive crack, $d$ the current length of the process zone and $G$ the energy release rate at the end of the loading phase of the cycle $N$, we have $\mathrm{d}=\epsilon D^{0}$ and $G=\epsilon \mathrm{G}[\ell]$. If we use the classical notations of engineers and denote by $\frac{d \ell}{d N}$ the increment of the length of the non cohesive crack during a cycle, we get $\frac{d \ell}{d N}=\epsilon \dot{\ell}$. Accordingly, (39) becomes

$\sum_{i=0}^{+\infty} V\left(\frac{i}{\mathrm{~d}} \frac{d \ell}{d N}\right) \frac{G}{G_{c}}=1 \quad$ with $\quad \mathrm{d}=\frac{\pi}{8\left(1-v^{2}\right)} \frac{E}{\sigma_{c}} \frac{G}{G_{c}} d_{c}$.

The properties of this equation already obtained in Sect. 3.3.5 can be rephrazed and reinterpreted as follows:

1. If $G>G_{c}$, then there is no solution for (42);

2. If $G=G_{c}$, then the solutions for (42) are all the $d \ell / d N$ greater than or equal to $\frac{\pi E d_{c}}{8\left(1-v^{2}\right) \sigma_{c}}$;

3. If $0<G<G_{c}$, then there exists a unique solution for (42) and it can read as

$$
\frac{d \ell}{d N}=\frac{\pi}{8\left(1-v^{2}\right)} \frac{E}{\sigma_{c}} \mathrm{f}\left(\frac{G}{G_{c}}\right) d_{c} .
$$

In other words, we obtain the same property as in Griffith's law: the energy release rate cannot be greater than $G_{c}$ and the growth rate of the crack is undetermined when the energy release rate is equal to $G_{c}$. That means that the propagation of the crack during a cycle is no more of the order of $d_{c}$ if the energy release rate becomes greater than $G_{c}$ at the end of the loading phase. The propagation is no more governed by fatigue concepts. That corresponds to situation where the propagation is "brutal", that is discontinuous in time. On the other hand, if the energy release rate at the end of the loading phase of a cycle is less than $G_{c}$, then the crack propagates progressively from one cycle to the other (whereas there is no propagation with Griffith's law). The presence of this subcritical regime is due to the introduction of cohesive forces and of the cumulated opening concept into the model.

Let us establish additional properties for the function $f$.

$\mathbf{P 1} \mathrm{f}$ is a dimensionless function, defined on $(0,1)$, continuously differentiable, increasing from 0 to 1, see Fig. 8.

P2 For small values of $g=G / G_{c}$, we have $\mathrm{f}(g)=$ $g^{2} / 3+o\left(g^{2}\right)$ and the fatigue law is like a Paris' law with exponent 4 in terms of the stress intensity factor: 


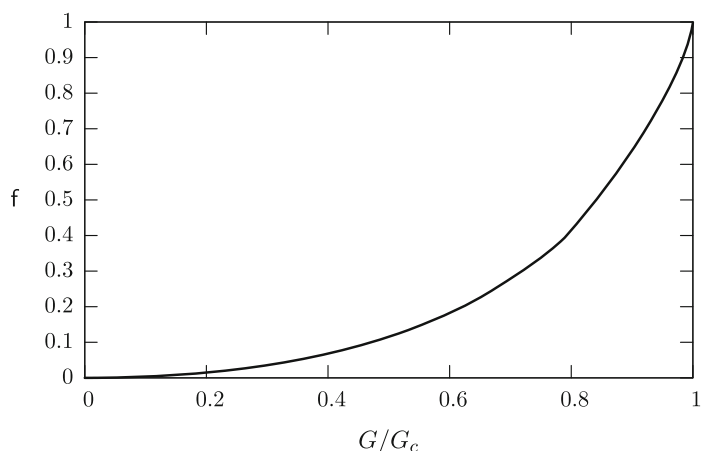

Fig. 8 Graph of the function $f$ giving the fatigue law

$\frac{d \ell}{d N} \approx \frac{\pi}{24\left(1-v^{2}\right)} \frac{E}{\sigma_{c}}\left(\frac{G}{G_{c}}\right)^{2} d_{c}$.

Let us prove P1. Setting $F(f, g)=-1+\sum_{i=0}^{+\infty} g V(i f / g)$ for $f \geq 0$ and $g \in(0,1]$, (42) reads then as $F(\mathrm{f}(g), g)=0$ with $g=G / G_{c}$. The regularity of $f$ on $(0,1)$ is a direct consequence of the implicit function theorem, $V$ being continuously differentiable and $V^{\prime} \neq 0$ on $(0,1)$. Since $F$ is an increasing function of $g$ at fixed $f$ and since $f \mapsto F(f, g)$ decreases from infinity to $-1+g<0$ when $f$ goes from 0 to $g, g \longmapsto \mathrm{f}(g)$ is increasing and $0<\mathrm{f}(g)<g$. Hence $\lim _{g \rightarrow 0} f(g)=0$. Since $F(f, 1)=\sum_{i=1}^{+\infty} V($ if $), F(f, 1)>0$ if $f<1$ and $F(f, 1)=0$ if $f \geq 1$. Hence $\lim _{g \rightarrow 1} \mathrm{f}(g)=1$ which completes P1.

Let us prove P2. We know by P1 that $\lim _{g \rightarrow 0} f(g)=0$. Let $n(g)$ be the integer part of $g / f(g)$. Since $\sum_{i=0}^{n(g)} g V($ if $(g) / g)=1$, we have $\lim _{g \rightarrow 0} n(g)=\infty$ and $\lim _{g \rightarrow 0} n(g) \mathrm{f}(g) / g=1$. Therefore

$\lim _{g \rightarrow 0} \frac{f(g)}{g^{2}}=\lim _{g \rightarrow 0} \sum_{i=0}^{n(g)} \frac{1}{n(g)} V\left(\frac{i}{n(g)}\right)=\int_{0}^{1} V(\zeta) d \zeta=\frac{1}{3}$

and the result follows.

The fatigue law depends on the choice of the surface energy density (here, Dugdale's law), on the choice of the memory variable and more generally of the irreversibility condition (here, the cumulated opening) and on the type of cyclic loading (here, mode I simple cycle). In the next subsections, we show what happens if one of these parameters changes.

\subsection{Influence of the type of cyclic loading: cycles} with partial unloading

Let us consider a cyclic loading with period 2 where the rescaled stress intensity factor of the large scale problem oscillates between $K_{I}^{m}>0$ and $K_{I}^{M}>K_{I}^{m}$, see Fig. 9. We can follow the same procedure as in the case of a simple cyclic loading to obtain the fatigue law. Let us simply give the

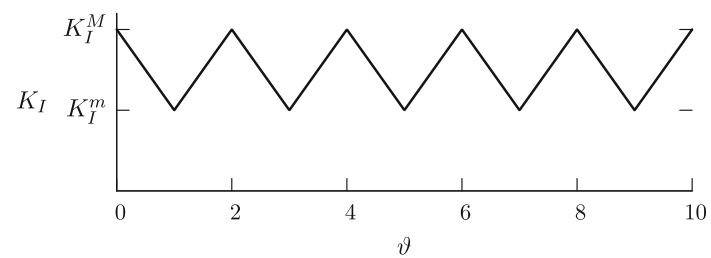

Fig. 9 Non simple cyclic loading: the rescaled stress intensity factor $\mathrm{K}_{\mathrm{I}}$ oscillates between $\mathrm{K}_{\mathrm{I}}^{\mathrm{m}}$ and $\mathrm{K}_{\mathrm{I}}^{\mathrm{M}}$ (the true one $K_{I}=\sqrt{\epsilon} \mathrm{K}_{\mathrm{I}}$ )

main steps by emphasizing the changes in comparison with the simple cycle case. All the process zone is active at the end of the loading phase, the solution of the small scale problem at $\vartheta=0$ is unchanged, $\llbracket U_{2}^{0} \rrbracket$ and $D^{0}$ are still given by (32) and (33) with $\mathrm{K}_{\mathrm{I}}=\mathrm{K}_{\mathrm{I}}^{\mathrm{M}}$ :

$D^{0}=\frac{\pi \mathrm{K}_{\mathrm{I}}^{\mathrm{M}}}{8 \sigma_{c}^{2}}=\frac{\pi E \mathrm{G}^{\mathrm{M}}}{8\left(1-v^{2}\right) \sigma_{c}^{2}}, \quad \llbracket U_{2}^{0} \rrbracket(x)=V\left(\frac{x}{D^{0}}\right) \frac{\mathrm{G}^{\mathrm{M}}}{\sigma_{c}}$.

At the end of the unloading phase, since $K_{\mathrm{I}}^{\mathrm{m}}>0$, only a part of the process zone is passive, the remaining part being neutral. The solution of the small scale problem at $\vartheta=1$ is the same as the one found in Sect. 3.3.3 at $\vartheta=1-\mathrm{K}_{\mathrm{I}}^{\mathrm{m}} / \mathrm{K}_{\mathrm{I}}^{\mathrm{M}}$ with $\mathrm{K}_{\mathrm{I}}=\mathrm{K}_{\mathrm{I}}^{\mathrm{M}}$. Therefore, we get

$\left[\left[U_{2}^{1}\right]\right](x)=\left(V\left(\frac{x}{D^{0}}\right)-(1-\kappa)^{2} V\left(\frac{x}{(1-\kappa)^{2} D^{0}}\right)\right) \frac{\mathrm{G}^{\mathrm{M}}}{\sigma_{c}}$

where $\kappa=\mathrm{K}_{\mathrm{I}}^{\mathrm{m}} / \mathrm{K}_{\mathrm{I}}^{\mathrm{M}}$. During the unloading phase the opening is non increasing, $\left[\left[\dot{U}_{2}^{\vartheta}\right]\right] \leq 0$, while during the loading phase the opening is non decreasing, $\left[\left[\dot{U}_{2}^{\vartheta}\right]\right] \geq 0$. Accordingly, the cumulated opening is such that

$$
\begin{aligned}
\Delta^{0}(x) & =\Delta^{-2}(x)+\int_{-2}^{0}\left(\llbracket \dot{U}_{2}^{\vartheta} \rrbracket(x)\right)^{+} d \vartheta \\
& =\Delta^{-2}(x)+\llbracket U_{2}^{0} \rrbracket(x)-\llbracket U_{2}^{-1} \rrbracket(x) \\
& =\Delta^{0}(x+\dot{\ell})+\llbracket U_{2}^{0} \rrbracket(x)-\llbracket U_{2}^{1} \rrbracket(x+\dot{\ell}) .
\end{aligned}
$$

By induction we obtain

$\Delta^{0}(x)=\sum_{i=0}^{\infty} \llbracket U_{2}^{0} \rrbracket(x+i \dot{\ell})-\sum_{i=1}^{\infty} \llbracket U_{2}^{1} \rrbracket(x+i \dot{\ell})$.

Using (45) and (46) the condition $\Delta^{0}(0)=L_{c}$ giving the equation for $\dot{\ell}$ becomes

$\sum_{i=1}^{\infty} V\left(\frac{i \dot{\ell}}{(1-\kappa)^{2} D^{0}}\right)(1-\kappa)^{2} \mathrm{G}^{\mathrm{M}}=\sigma_{c} L_{c}-\mathrm{G}^{\mathrm{M}}$.

Reintroducing the true physical quantities, we finally obtain the following equation for $d \ell / d N$ :

$\sum_{i=0}^{\infty} V\left(\frac{i}{(1-\kappa)^{2} \mathrm{~d}} \frac{d \ell}{d N}\right)(1-\kappa)^{2} \frac{G^{M}}{G_{c}}=1-\left(2 \kappa-\kappa^{2}\right) \frac{G^{M}}{G_{c}}$ 
where

$$
\begin{aligned}
& \mathrm{d}=\frac{\pi}{8\left(1-v^{2}\right)} \frac{E}{\sigma_{c}} \frac{G^{M}}{G_{c}} d_{c}, \quad G^{M}=\left(1-v^{2}\right) \frac{K_{I}^{M^{2}}}{E} \\
& \kappa=\frac{K_{I}^{m}}{K_{I}^{M}}
\end{aligned}
$$

$K_{I}^{m}$ and $K_{I}^{M}$ being the real stress intensity factors at the end of the loading phase and the unloading phase, respectively.

As is the case of a simple cycle, this equation admits a unique (positive) solution if and only if $0<G^{M}<G_{c}$. Now, the solution $d \ell / d N$ depends both on the maximal energy release rate $G^{M}$ and on $\Delta K_{I}=K_{I}^{M}-K_{I}^{m}$. Specifically, using the function $f$ defined in (43) we get

$$
\begin{aligned}
\frac{d \ell}{d N}= & \frac{\pi}{8\left(1-v^{2}\right)} \frac{E}{\sigma_{c}} \frac{G_{c}}{G_{c}-\left(2 \kappa-\kappa^{2}\right) G^{M}} \\
& \times \mathrm{f}\left(\frac{(1-\kappa)^{2} G^{M}}{G_{c}-\left(2 \kappa-\kappa^{2}\right) G^{M}}\right) d_{c} .
\end{aligned}
$$

When the amplitude of the cycle is small, i.e., when $\kappa$ is close to 1 , we obtain

$\frac{d \ell}{d N} \approx \frac{\pi}{24\left(1-v^{2}\right)} \frac{E}{\sigma_{c}} \frac{\left(\Delta K_{I}\right)^{4} K_{I c}^{2}}{\left(K_{I c}^{2}-\left(K_{I}^{M}\right)^{2}\right)^{3}} d_{c}$

where $K_{I c}$ denotes the toughness, i.e., $G_{c}=\left(1-v^{2}\right) K_{I c}^{2} / E$. The dependence on the amplitude and on the maximal value of the stress intensity factor is explicit. If, furthermore, $K_{I}^{M}$ is small in comparison with $K_{I c}$, we recover the usual Paris' law with the exponent 4, see [34]:

$\frac{d \ell}{d N} \approx \frac{\pi}{24\left(1-v^{2}\right)} \frac{E}{\sigma_{c}}\left(\frac{\Delta K_{I}}{K_{I c}}\right)^{4} d_{c}$.

\subsection{Influence of the memory variable: cumulated tearing in mode III}

In mode III, since there is no opening, the non interpenetration condition is automatically satisfied but the cumulated opening is no more a memory variable. A possible candidate is the cumulated tearing

$\delta^{t}(s)=\int_{0}^{t}\left|\llbracket \dot{u}_{3}^{t^{\prime}} \rrbracket\right|(s) d t^{\prime}$

where $u_{3}^{t}$ denotes the anti-plane component of the displacement field at time $t$. Thus, the cumulated tearing evolves as soon as the anti-plane displacement changes, whatever the sign of its rate. Let us still consider Dugdale's surface energy density $\phi(\delta)=\min \left\{\tau_{c} \delta, G_{c}\right\}$ where $\tau_{c}$ denotes the critical shear stress and $\tau=\sigma_{3 i} n_{i}$ is the shear stress on the crack lip. Following the same variational procedure as in Sect. 2.2, the shear stress-cumulated tearing conditions read as $\left\{\begin{array}{lll}\left|\tau^{t}\right| \leq \tau_{c} & \text { on } & \Gamma_{\mathrm{B}}^{t} \\ \tau^{t}=0 & \text { on } & \Gamma_{\mathrm{D}}^{t}\end{array}\right.$,
$\left\{\begin{array}{lll}\tau^{t}=\operatorname{sign}\left(\llbracket \dot{u}_{3}^{t} \rrbracket\right) \tau_{c} & \text { on } & \Gamma_{\mathrm{Ca}}^{t} \\ \left|\tau^{t}\right| \leq \tau_{c} & \text { on } & \Gamma_{\mathrm{Cn}}^{t}\end{array}\right.$

with

$\left\{\begin{array}{c}\Gamma_{\mathrm{B}}^{t}=\left\{s \in \hat{\Gamma}: \delta^{t}(s)=0\right\} \\ \Gamma_{\mathrm{D}}^{t}=\left\{s \in \hat{\Gamma}: \delta^{t}(s)>d_{c}\right\}\end{array}\right.$,

$\left\{\begin{array}{l}\Gamma_{\mathrm{Ca}}^{t}=\left\{s \in \hat{\Gamma}: 0<\delta^{t}(s)<d_{c}, \llbracket \dot{u}_{3}^{t} \rrbracket(s) \neq 0\right\} \\ \Gamma_{\mathrm{Cn}}^{t}=\left\{s \in \hat{\Gamma}: 0<\delta^{t}(s)<d_{c}, \llbracket \dot{u}_{3}^{t} \rrbracket(s)=0\right\}\end{array}\right.$

and $d_{c}=G_{c} / \tau_{c}$. There does not exist a passive zone inside the process zone. Let us consider symmetric cyclic loading i.e. a proportional loading such that the seesaw function $\varpi$ is the periodic function with period 2 defined on $[-1,1]$ by $\varpi(t)=1-2|t|$, see Fig. 10. (Figs. 11 and 12)

Assuming that $d_{c} \ll L_{c}$, we can again follow the twoscale approach to obtain the fatigue law giving the growth rate of the non cohesive crack $d \ell / d N$ at each cycle in terms of the maximal mode III stress intensity factor $K_{I I I}$ (or equivalently the maximal energy release rate during the cycle). We simply report here the main results, see [2] for details. The size $d$ of the process zone $\Gamma_{C}$ is such that there is no singularity at the tip of the process zone, $d$ is of the order of $d_{c}$ and given by

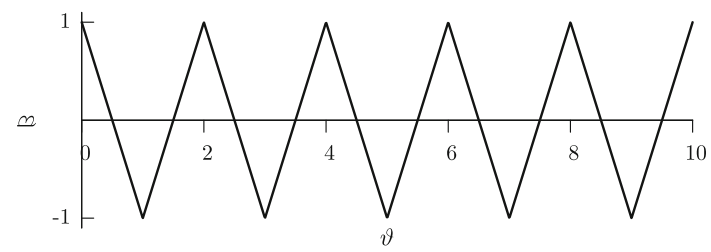

Fig. 10 Symmetric cyclic loading used in mode III

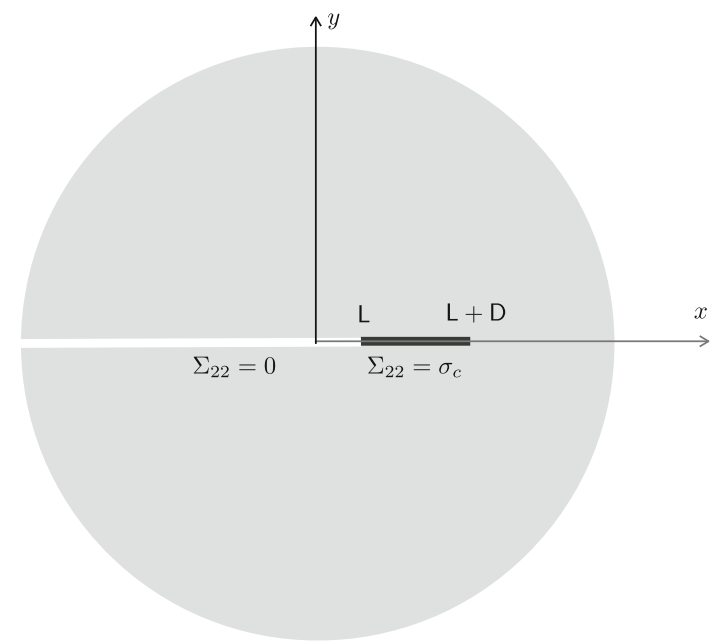

Fig. 11 Data of the generic small scale problem 
Fig. 12 Graphs of the normal stress and of the function $V$ giving the jump of the normal displacement on the crack path
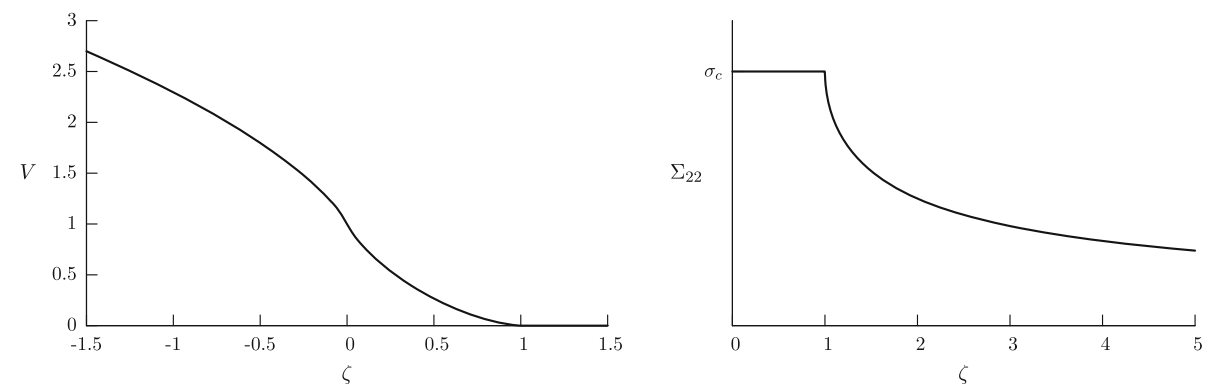

$\mathrm{d}=\frac{\pi}{4} \frac{G}{G_{c}} \frac{\mu}{\tau_{c}} d_{c}$ with $\quad G=\frac{K_{I I}^{2}}{2 \mu}$.

The cumulated tearing is equal to $d_{c}$ at the tip of the non cohesive crack $\Gamma_{\mathrm{D}}$. That yields the equation for $d \ell / d N$ which reads as

$1-\frac{G}{G_{c}}=\frac{G}{G_{c}} \sum_{i=1}^{\infty} 2 V\left(\frac{i}{2 \mathrm{~d}} \frac{d \ell}{d N}\right)$

where $V$ is still given by (55). This equation enjoys the same properties as (43): there exists no solution if $G>G_{c}$, an infinite number of solutions if $G=G_{c}$ and a unique solution if $0<G<G_{c}$. In this latter case the solution can read as

$\frac{d \ell}{d N}=\frac{\pi}{4} \frac{\mu}{\tau_{c}}\left(1+\frac{G}{G_{c}}\right) \mathrm{f}\left(\frac{2 G}{G_{c}+G}\right) d_{c}$

where $\mathrm{f}$ is the function defined in (43). When $G / G_{c}$ is small, we recover a Paris law with the exponent 4 in terms of the stress intensity factor:

$\frac{d \ell}{d N} \approx \frac{\pi}{3} \frac{\mu}{\tau_{c}}\left(\frac{G}{G_{c}}\right)^{2} d_{c}$.

The constant multiplier differs from that of the mode I case with simple cyclic loading.

\subsection{Influence of the surface energy density}

If we replace Dugdale's surface energy density by a more general, Barenblatt's type, surface energy density, we loose the advantage of explicitely constructing the fatigue law. Indeed, in general, it is no more possible to obtain the solution of the small scale problem in a closed form. Moreover the concept of non cohesive crack is not necessarily meaningful, at least at a small scale. Indeed if $\phi$ is always increasing with $\phi^{\prime}>0$, then the cohesive forces never vanish. It is the case for example when $\phi$ $(\delta)=G_{c}\left(1-\exp \left(-\delta / d_{c}\right)\right)$. In such a case, the two-scale approach must be refined. This is outside the scope of the present paper and will the subject of future works, see however [1] and [19] for some preliminary results in this context.

\section{Concluding remarks and perspectives}

The construction of the fatigue law proposed in Sect. 3 is based on a separation of scales in space and time which is licit provided that the characteristic length of the material is small by comparison to any characteristic length of the body. The small scale problem consists in determining the stationary regime governing the evolution of the crack and of the process zone during one cycle of loading with the effective stress intensity factor as the loading parameter. This problem has a universal character since it depends neither on the geometry of the body, neither of the crack path nor of the boundary conditions. Therefore the resulting fatigue law is characteristic of the material properties (including its bulk behavior and the cohesive model) for a given type of cyclic loading. In the case of a linear elastic material with a Dugdale-type surface energy depending only on the cumulated opening, we have obtained a Paristype fatigue law which is approximatively a power law like $d \ell / d N=C \Delta \mathrm{K}_{\mathrm{I}}^{4}$ for small values of $\Delta \mathrm{K}_{\mathrm{I}}$. An interesting task will be to understand the origin of this exponent 4 and to obtain other exponents by changing the surface energy, the loading-unloading condition or the bulk behavior of the material. Another important issue is to see how one could account for the concept of fatigue threshold from cohesive models. Among all possible developments of our approach, let us cite the most exciting: (1) to study the nucleation of a crack by fatigue; (2) to study the effect of an overloading; (3) to study the influence of different sequences of loading; (4) to construct fatigue law under mixed mode conditions.

\section{Appendix 1: The generic problem in the neighborhood of the crack tip}

The plane is equipped with the cartesian coordinate system $(x, y)$, the associated canonical basis is $(\mathbf{i}, \mathbf{j})$ and $z=x+i y$ denotes the affixe of the complex number associated with the point $(x, y)$. Let $\mathrm{K}, \mathrm{L}$ and $\mathrm{D}$ be three given real numbers with $\mathrm{K}>0$ and $\mathrm{D}>0$. Let us consider the following planestrain elastic problem whose unknowns are the displacement and stress field $\mathbf{U}$ and $\boldsymbol{\Sigma}$ : 


$$
\begin{aligned}
& \operatorname{div} \boldsymbol{\Sigma}=0, \quad \boldsymbol{\Sigma}=\lambda \operatorname{div} \mathbf{U I}+2 \mu \boldsymbol{\varepsilon}(\mathbf{U}) \\
& \text { in } \quad \mathbb{R}^{2} \backslash(-\infty, \mathrm{L}+\mathrm{D}) \times\{0\}, \\
& \Sigma_{12}=\Sigma_{22}=0 \quad \text { on } \quad(-\infty, \mathrm{L}) \times\{0\}, \\
& \Sigma_{12}=0, \Sigma_{22}=\sigma_{c} \quad \text { on } \quad(\mathrm{L}, \mathrm{L}+\mathrm{D}) \times\{0\},
\end{aligned}
$$

with the condition at infinity

$\lim _{r \rightarrow \infty}\left(\mathbf{U}(x, y)-\frac{\mathrm{K}}{2 \mu} \sqrt{\frac{r}{2 \pi}} \mathbf{u}^{S}(\theta)\right)=0$

where $x=r \cos \theta, y=r \sin \theta$ and $\mathbf{u}^{S}(\theta)=(3-4 v-\cos \theta)$ $\left(\cos \frac{\theta}{2} \mathbf{i}+\sin \frac{\theta}{2} \mathbf{j}\right)$. This problem admits a unique solution which can be obtained in a closed form by using the theory of complex potentials, cf [26]. We simply recall here the main results. The fields $\mathbf{U}$ et $\boldsymbol{\Sigma}$ are related to the function $\varphi(z)$ of the complex variable $z$ by

$$
\begin{aligned}
& \Sigma_{22}(x, y)-\mathrm{i} \Sigma_{12}(x, y)=\varphi^{\prime}(z)+\varphi^{\prime}(\bar{z})+(z-\bar{z}) \overline{\varphi^{\prime \prime}(z)}, \\
& 2 \mu\left(U_{1}(x, y)+\mathrm{i} U_{2}(x, y)\right)=(3-4 v) \varphi(z)-\varphi(\bar{z})-(z-\bar{z}) \overline{\varphi^{\prime}(z)},
\end{aligned}
$$

$\varphi$ being holomorphic in the plane without the half-line $(-\infty, \mathrm{L}+\mathrm{D}) \times\{0\}$, the bar denoting the complex conjugate. By a standard procedure, we get

$$
\begin{aligned}
\varphi^{\prime}(z)= & \frac{\sigma_{c}}{2 \pi \sqrt{z-\mathrm{L}-\mathrm{D}}} \int_{\mathrm{L}}^{\mathrm{L}+\mathrm{D}} \frac{\sqrt{\mathrm{L}+\mathrm{D}-x}}{x-z} d x \\
& +\frac{\mathrm{K}}{2 \sqrt{2 \pi(z-\mathrm{L}-\mathrm{D})}} .
\end{aligned}
$$

Near the tip $z=\mathrm{L}+\mathrm{D}, \varphi^{\prime}(z)$ behaves like

$\varphi^{\prime}(z) \approx \frac{\sqrt{2 \pi} \mathrm{K}-4 \sigma_{c} \sqrt{\mathrm{D}}}{4 \pi \sqrt{z-\mathrm{L}-\mathrm{D}}}$

and hence the stresses are singular with the usual singularity in $1 / \sqrt{r}$ except if the factor $\sqrt{2 \pi} \mathrm{K}-4 \sigma_{c} \sqrt{\mathrm{D}}$ vanishes. Specifically, the jump of the normal displacement just behind the tip $x=\mathrm{L}+\mathrm{D}$ and the normal stress just ahead the tip read as

$$
\begin{aligned}
& \llbracket U_{2} \rrbracket(r)=\frac{2\left(1-v^{2}\right)}{\pi E}\left(\sqrt{2 \pi} \mathrm{K}-4 \sigma_{c} \sqrt{\mathrm{D}}\right) \sqrt{r}+\cdots, \\
& \Sigma_{22}(r)=\frac{\sqrt{2 \pi} \mathrm{K}-4 \sigma_{c} \sqrt{\mathrm{D}}}{4 \pi \sqrt{r}}+\cdots .
\end{aligned}
$$

Therefore, if it is required that $\Sigma_{22} \leq \sigma_{c}$ on the half-line $(\mathrm{L}+\mathrm{D},+\infty) \times\{0\}$, then $\mathrm{K}$ and $\mathrm{D}$ must be such that $\sqrt{2 \pi} \mathrm{K} \leq 4 \sigma_{c} \sqrt{\mathrm{D}}$. On the other hand, if it is required that $\llbracket U_{2} \rrbracket \geq 0$ holds everywhere (by a non interpenetration condition, for instance), then $\mathrm{K}$ and $\mathrm{D}$ must satisfy the converse inequality $\sqrt{2 \pi} \mathrm{K} \geq 4 \sigma_{c} \sqrt{\mathrm{D}}$. Accordingly, in order that both conditions are satisfied, the solution must be non singular at the tip L + D. In such a case D and $\mathrm{K}$ are related by
$\mathrm{K}=4 \sigma_{c} \sqrt{\frac{\mathrm{D}}{2 \pi}}$.

Assuming from now on that (52) holds, (51) becomes

$$
\begin{aligned}
\varphi^{\prime}(z)= & \frac{\sigma_{c}}{2}+\frac{i \sigma_{c}}{2 \pi}(\log (\sqrt{\mathrm{D}}+i \sqrt{z-\mathrm{L}-\mathrm{D}}) \\
& -\log (\sqrt{\mathrm{D}}-i \sqrt{z-\mathrm{L}-\mathrm{D}})) .
\end{aligned}
$$

In (53), Log denotes the principal determination of the complex logarithm. After some calculations, one obtains that the normal jump of the displacement along the $x$-axis reads as

$\llbracket U_{2} \rrbracket(x)=V\left(\frac{x-\mathrm{L}}{\mathrm{D}}\right) \frac{8\left(1-v^{2}\right)}{\pi} \frac{\sigma_{c}}{E} \mathrm{D}$,

where $V$ denotes the dimensionless real-valued function defined by

$V(\zeta)= \begin{cases}\sqrt{1-\zeta}-\zeta \ln (1+\sqrt{1-\zeta})+\zeta \ln \sqrt{|\zeta|} & \text { if } \zeta \leq 1, \zeta \neq 0 \\ 0 & \text { if } \zeta \geq 1\end{cases}$

and $V(0)=1$. Let us note that $V$ is continuously differentiable everywhere (even at $\zeta=0$ and $\zeta=1$ ), is concave for $\zeta \leq 0$ and is strictly decreasing from $\infty$ to 0 when $\zeta$ goes from $-\infty$ to 1 . When $\zeta \rightarrow-\infty$, $V(\zeta)=2 \sqrt{|\zeta|}+o(1)$. The non interpenetration condition $\llbracket\left[U_{2} \rrbracket \geq 0\right.$ is satisfied everywhere. The normal stress $\Sigma_{22}$ along the half-line $(\mathrm{L}+\mathrm{D},+\infty) \times\{0\}$ is given by

$\Sigma_{22}(x, 0)=\left(1-\frac{2}{\pi} \arcsin \sqrt{1-\frac{\mathrm{D}}{x-\mathrm{L}}}\right) \sigma_{c}$.

It decreases from $\sigma_{c}$ to 0 and, therefore, the condition $\Sigma_{22} \leq \sigma_{c}$ is satisfied.

\section{References}

1. Abdelmoula R, Marigo J-J, Weller T (2009) Construction des lois de fatigue à partir de modèles de forces cohésives: cas de fissures en mode I. Comptes Rendus Mécanique 337(3):166-172

2. Abdelmoula R, Marigo J-J, Weller T (2009) Construction d'une loi de fatigue à partir d'un modèle de forces cohésives: cas d'une fissure en mode III. Comptes Rendus Mécanique 337(1):53-59

3. Abdul-Baqi A, Schreurs PJG, Geers MGD (2005) Fatigue damage modeling in solder interconnects using a cohesive zone approach. Int J Sol Struct 42:927-942

4. Barenblatt GI (1962) The mathematical theory of equilibrium cracks in brittle fracture. Adv Appl Mech 55-129

5. Bourdin B, Francfort GA, Marigo J-J (2008) The variational approach to fracture. J Elast 91(1-3):5-148

6. Bui HD (1978) Mécanique de la rupture fragile. Masson

7. Charlotte M, Laverne J, Marigo J-J (2006) Initiation of cracks with cohesive force models: a variational approach. Eur J Mech A-Solid 25(4):649-669 
8. Cherepanov GP (1979) Mechanics of brittle fracture. McGrawHill International Book Company, London

9. Dal Maso G, Francfort GA, Toader R (2005) Quasistatic crack growth in nonlinear elasticity. Arch Ration Mech Anal 176(2):165-225

10. Dal Maso G, Toader R (2002) A model for the quasi-static growth of brittle fractures: existence and approximation results. Arch Ration Mech Anal 162:101-135

11. Dugdale DS (1960) Yielding of steel sheets containing slits. J Mech Phys Solids 8:100-108

12. Ferdjani H, Abdelmoula R, Marigo J-J (2007) Insensitivity to small defects of the rupture of materials governed by the Dugdale model. Continuum Mech Therm 19(3-4):191-210

13. Ferriero A (2008) Quasi-static evolution for fatigue debonding. ESAIM Control Optim Calc Var 4(2):233-253

14. Francfort GA, Marigo J-J (1998) Revisiting brittle fracture as an energy minimization problem. J Mech Phys Solids 46(8): 1319-1342

15. Francfort GA, Mielke A (2006) Existence results for a class of rate-independent material models with nonconvex elastic energies. J Reine Angew Math 595:55-91

16. Giacomini A (2005) Size effects on quasi-static growth of cracks. SIAM J Math Anal 36(6):1887-1928 (electronic)

17. Griffith A (1920) The phenomena of rupture and flow in solids. Phil Trans Roy Soc Lond CCXXI(A):163-198

18. Jaubert A, Marigo J-J (2005) L'approche variationnelle de la fatigue : des premiers résultats. Comptes Rendus Mécanique 333(7):550-556

19. Jaubert A, Marigo J-J (2006) Justification of Paris-type fatigue laws from cohesive forces model via a variational approach. Continuum Mech Therm V18(1):23-45

20. Laverne J, Marigo J-J (2004) Global approach, relative minima and yield criterion in fracture mechanics. Comptes Rendus Mécanique 332(4):313-318

21. Lawn B (1993) Fracture of brittle solids. Cambridge solid state science series, 2nd edn. Cambridge University press, Cambridge
22. Maiti S, Geubelle P (2005) A cohesive model for fatigue failure of polymers. Eng Fract Mech 72(5):691-708

23. Marigo J-J (1985) Modelling of brittle and fatigue damage for elastic material by growth of microvoids. Eng Fract Mech 21(4):861-874

24. Marigo J-J, Truskinovky L (2004) Initiation and propagation of fracture in the models of Griffith and Barenblatt. Continuum Mech Therm 16(4):391-409

25. Mielke A (2005) Evolution of rate-independent systems. In: Evolutionary equations, volume II of Handb Differ Equ. Elsevier/ North-Holland, Amsterdam, pp 461-559

26. Muskhelishvili NI (1953) Some basic problems of mathematical theory of elasticity, 2nd edn. P. Noordhoff Ltd, Groningen

27. Needleman A (1992) Micromechanical modelling of interface decohesion. Ultramicroscopy 40:203-214

28. Nguyen O, Repetto EA, Ortiz M, Radovitzki RA (2001) A cohesive model of fatigue crack growth. Int J Fract 110:351-369

29. Nguyen QS (2000) Stability and nonlinear solid mechanics. Wiley, London

30. Paris PC, Erdogan F (1963) A critical analysis of crack propagation laws. J Basic Eng 85:528-534

31. Paris PC, Gomez MP, Anderson WE (1961) A rational analytic theory of fatigue. Trend Eng 13(8):9-14

32. Roe KL, Siegmund T (2002) An irreversible cohesive zone model for interface fatigue crack growth simulation. Eng Fract Mech 70:209-232

33. Siegmund T (2004) A numerical study of transient fatigue crack growth by use of an irreversible cohesive zone model. Int $\mathrm{J}$ Fatigue 26(9):929-939

34. Suresh S (1998) Fatigue of materials. Cambridge Editions, Cambridge

35. Weertman J (1966) Rate of growth of fatigue cracks calculated from the theory of infinitesimal dislocations distributed on a plane. Int J Frac Mech 2:460-467

36. Yang B, Mall S, Ravi-Chandar K (1999) A cohesive zone model for fatigue crack growth in quasibrittle materials. Int J Sol Struct 38:3927-3944 